The Astrophysical Journal, 673:418-433, 2008 January 20

Copyright is not claimed for this article. Printed in U.S.A.

\title{
THE KECK APERTURE MASKING EXPERIMENT: MULTIWAVELENGTH OBSERVATIONS OF SIX MIRA VARIABLES
}

\author{
H. C. Woodruff, ${ }^{1}$ P. G. Tuthill,${ }^{1}$ J. D. Monnier, ${ }^{2}$ M. J. Ireland,${ }^{3}$ T. R. Bedding, ${ }^{1}$ \\ S. Lacour, ${ }^{1}$ W. C. Danchi, ${ }^{4}$ and M. SchOlz ${ }^{1,5}$ \\ Received 2007 July 30; accepted 2007 September 24
}

\begin{abstract}
The angular diameters of six oxygen-rich Mira-type long-period variables have been measured at various NIR wavelengths using the aperture-masking technique in an extensive observing program from 1997 January to 2004 September. These data sets span many pulsation cycles of the observed objects and represent the largest study of multiwavelength, multiepoch interferometric angular diameter measurements on Mira stars to date. The calibrated visibility data of $o$ Cet, R Leo, R Cas, W Hya, $\chi$ Cyg, and R Hya are fitted using a uniform disk brightness distribution model to facilitate comparison between epochs, wavelengths, and with existing data and theoretical models. The variation of angular diameter as a function of wavelength and time is studied, and cyclic diameter variations are detected for all objects in our sample. These variations are believed to stem from time-dependent changes of density and temperature (and hence varying molecular opacities) in different layers of these stars. The similarities and differences in behavior between these objects are analyzed and discussed in the context of existing theoretical models. Furthermore, we present time-dependent $3.08 \mu \mathrm{m}$ angular diameter measurements, probing for the first time these zones of probable dust formation, which show unforeseen sizes and are consistently out of phase with other NIR layers shown in this study. The S-type Mira $\chi$ Cyg exhibits significantly different behavior compared to the M-type Mira variables in this study, in both its NIR light curves and its diameter pulsation signature. Our data show that the NIR diameters predicted by current models are too small and need to incorporate additional and/or enhanced opacity mechanisms. Also, new tailored models are needed to explain the behavior of the S-type Mira $\chi$ Cyg.
\end{abstract}

Subject headings: instrumentation: interferometers - stars: AGB and post-AGB — stars: fundamental parameters stars: individual (Mira, R Hya, Chi Cyg, W Hya, R Leo, R Cas) — stars: late-type techniques: interferometric

\section{INTRODUCTION}

Mira variables are pulsating M-type giants with very extended stellar atmosphere and mass-loss rates of up to $10^{-4} M_{\odot} \mathrm{yr}^{-1}$ (e.g., Jura \& Kleinmann 1990). Multiwavelength studies allow us to probe the atmospheric structure, including $\mathrm{H}_{2} \mathrm{O}$ and dust shells. Optical and near-infrared (NIR) interferometry has been able to constrain fundamental parameters such as intensity distributions, effective temperatures and diameters, and the dependence of these on wavelength and pulsation phase (e.g., Haniff et al. 1995; van Belle et al. 1996; Perrin et al. 1999; Young et al. 2000; Hofmann et al. 2002; Thompson et al. 2002; Woodruff et al. 2004). Interferometric studies of Mira stars conducted over multiple epochs and spanning a range of wavelengths have helped to address fundamental questions such as the pulsation mode of these stars (e.g., Woodruff et al. 2004; Fedele et al. 2005), the molecular and dust abundances in the atmosphere (e.g., Ireland et al. 2004a, 2004b; Ireland \& Scholz 2006), the characteristics of the circumstellar environment (e.g., Danchi et al. 1994), and photospheric/circumstellar asymmetries (e.g., Ragland et al. 2006).

Interferometry, together with a host of other observational techniques, has gradually been making advances into our understand-

\footnotetext{
${ }^{1}$ School of Physics, University of Sydney, Sydney NSW 2006, Australia.

2 Department of Astronomy, University of Michigan at Ann Arbor, Ann Arbor, MI 48109-1090.

3 Planetary Science, California Institute of Technology, Pasadena, CA 91125.

${ }^{4}$ NASA Goddard Space Flight Center, Infrared Astrophysics, Code 685, Greenbelt, MD 20771.

${ }_{5}$ Institut für Theoretische Astrophysik der Universität Heidelberg, 69120 Heidelberg, Germany.
}

ing of the basic physics of these stars. For example, there is now consensus that Mira variables pulsate in the fundamental mode, based on photometry, e.g., MACHO observations (see Wood et al. 1999), and spectroscopy (see Scholz \& Wood 2000) in addition to interferometry. However, the structure and composition of the stellar atmosphere still show surprising results when investigated in detail. Ragland et al. (2006) have, e.g., detected $H$-band asymmetric brightness distributions in about $29 \%$ of their sample of nearby asymptotic giant branch stars, substantiating another level of complexity in the structure of this class of objects.

In this paper we present a comprehensive interferometric study of Mira variables, encompassing six nearby objects, observed at up to 19 different phases in four filters. With this homogeneous data set, we are able to examine phase-dependent variations in Mira variables' atmospheric structure and are in a position to investigate long-term effects that span several pulsation cycles. By observing in the NIR (especially within the $J, H$, and $K$ bandpasses), we can sample molecular strata near the continuumforming layers that are often close to the position of the Rosseland layer $\left(\tau_{\text {Ross }}=1\right.$; cf. Scholz 2003 and references therein).

Theoretical models describe the molecular layering in the atmosphere and its variation with time (e.g., Bessell et al. 1996; Hofmann et al. 1998; Ireland et al. 2004a, 2004b) and make predictions of observables such as light curves, diameters, and intensity distributions. With our extensive database we can challenge existing models and motivate the development of a more complete physical picture of Mira variables. Ultimately, the aim of interferometric observations like these is to calibrate the theoretical models so that fundamental parameters of Mira variables can be 
TABLE 1

OBserved OBJects

\begin{tabular}{cccrc}
\hline \hline Name & $\begin{array}{c}\text { Period } \\
(\text { days })\end{array}$ & Spectral Type Range & $\begin{array}{c}\text { Distance } \\
(\mathrm{pc})\end{array}$ & $\begin{array}{c}\text { Distance } \\
\text { Reference }\end{array}$ \\
\hline${ }_{\text {o Cet............. }}$ & 332 & M5-9e & $107 \pm 6$ & 1 \\
R Leo ........... & 312 & M6-9.5e & $82 \pm 5$ & 1 \\
R Cas........... & 430 & M6-10e & $100 \pm 5$ & 2 \\
W Hya.......... & 385 & M7.5-9ep & $78 \pm 3$ & 1 \\
$\chi$ Cyg ........... & 408 & S6-S9(MSe) & $149 \pm 11$ & 1 \\
R Hya........... & 380 & M6-9eS(Tc) & $118 \pm 7$ & 1 \\
\hline
\end{tabular}

Notes.-Object period from the American Association of Variable Star Observers (AAVSO) visual light curves (A. A. Henden et al. 2006, private communication), with a periodicity error of $\approx 3 \%$ (see, e.g., Percy \& Au 1999), M spectral type range from Sloan \& Price (1998), and $\chi$ Cyg spectral type from Keenan \& Boeshaar (1980)

References.- (1) Knapp et al. 2003. (2) Vizier Online Data Catalog, J/A+A/ 399/1167 (D. Pourbaix et al., 2002).

derived from simple observables, enabling studies of stellar populations beyond the solar neighborhood.

\section{OBSERVATIONS AND DATA REDUCTION}

\subsection{Aperture-masking Observations}

Our sample of six Mira variables contains only nearby objects, all of which have revised Hipparcos distances (see Table 1). The objects were chosen for their large angular diameters and NIR brightness. Observations were performed with the $10 \mathrm{~m} \mathrm{Keck} \mathrm{I}$ telescope at a range of NIR wavelengths (see Table 2) using the Near Infrared Camera (NIRC). The telescope pupil was converted into a sparse interferometric array by placing aperture masks in the beam in front of the infrared secondary mirror, allowing the recovery of the Fourier amplitudes and closure phases for baselines up to $9.8 \mathrm{~m}$. For a detailed discussion of mask design, observing methodology, scientific rationale, and implementation, we refer to Tuthill et al. (2000b).

For this work we used nonredundant masks with 15 or 21 holes configured to deliver near-optimal sampling of the Fourier plane (Golay 1971). Data sets consisting of 100140 ms exposures were taken, alternating between the target of interest and nearby calibrator stars. The latter were chosen to have well-characterized, smaller apparent sizes (see Table 3). The data were recorded at 19 different epochs spanning more than $7 \mathrm{yr}$, delivering good coverage through the pulsation cycles of the objects observed. Tables 4-9 list these observations.

\subsection{Extraction of Visibilities}

The procedures for extracting the visibility amplitudes, as well as engineering and performance details, are documented in Monnier (1999) and Tuthill et al. (2000b), while recent scientific

TABLE 2

TABLE OF FiLTERS

\begin{tabular}{cccc}
\hline \hline \multicolumn{1}{c}{ Filter } & Keck/NIRC Filter Name & $\begin{array}{c}\text { Center Wavelength } \\
(\mu \mathrm{m})\end{array}$ & $\begin{array}{c}\text { Bandwidth } \\
(\mu \mathrm{m})\end{array}$ \\
\hline$z 1.08 \ldots \ldots \ldots .$. & HeI & 1.083 & 0.014 \\
$J 1.24 \ldots \ldots \ldots$. & OII & 1.236 & 0.011 \\
$H 1.65 \ldots \ldots \ldots$. & FeII & 1.647 & 0.018 \\
$K 2.26 \ldots \ldots \ldots$. & Kcont & 2.260 & 0.050 \\
& H221 & 2.261 & 0.0239 \\
$L 3.08 \ldots \ldots \ldots$. & PAHcs & 3.082 & 0.101 \\
$L 3.31 \ldots \ldots \ldots$. & PAH & 3.310 & 0.063 \\
\hline
\end{tabular}

TABLE 3

Calibrator Stars with Estimated Diameters

\begin{tabular}{|c|c|c|c|}
\hline Calibrator & Spectral Type & $\begin{array}{l}\text { Adopted UD Angular Diameter } \\
\text { (mas) }\end{array}$ & Reference \\
\hline$\alpha$ Cet ........... & M1.5 III & $11.7 \pm 0.6$ & 1 \\
\hline$\alpha$ Ari ........... & K2 III & $5.9 \pm 0.6$ & 1 \\
\hline$\alpha$ Cas........... & K0 III & $5.3 \pm 0.1$ & 2 \\
\hline$\beta$ Cet ........... & K0 III & $6.7 \pm 0.5$ & 3 \\
\hline$\alpha$ Нyа ........... & K3 II-III & $9.1 \pm 0.1$ & 4 \\
\hline$\pi$ Leo............ & M2 III & $4.6 \pm 0.3$ & 5 \\
\hline$\alpha \operatorname{Lyn} . . . \ldots \ldots . . .$. & K7 III & $7.2 \pm 0.6$ & 1 \\
\hline 2 Cen............ & M4.5 III & 14.7 & 6 \\
\hline$\gamma$ Sge $\ldots \ldots \ldots . . . .$. & M0 III & $6.0 \pm 0.6$ & 1 \\
\hline$\delta \operatorname{Sgr} \ldots \ldots \ldots . . . .$. & K3 III & $6.9 \pm 0.9$ & 7 \\
\hline 4 Cas ............ & M1 III & 4.3 & 6 \\
\hline$\epsilon \operatorname{Cyg} \ldots \ldots \ldots . . .$. & K0 III & $4.3 \pm 0.4$ & 2 \\
\hline$\delta \mathrm{Oph} \ldots \ldots . .$. & M0.5 III & $10.1 \pm 0.5$ & 8 \\
\hline Vega................... & A0 V & $3.203 \pm 0.003$ & 9 \\
\hline$\xi$ Cyg........... & K4.5 I & $7.5 \pm 0.6$ & 10 \\
\hline$\gamma$ Нyа ........... & G8 III & $3.0 \pm 0.2$ & 11 \\
\hline$\pi$ Нyа .......... & K2 III & $3.9 \pm 0.2$ & 3 \\
\hline
\end{tabular}

References.- (1) Dyck et al. 1998; (2) Mozurkewich et al. 1991; (3) CHARM catalog (Richichi \& Percheron 2002); (4) Mozurkewich et al. 2003; (5) Ridgway et al. 1979; (6) Dumm \& Schild 1998; (7) Monnier et al. 2004; (8) Perrin et al. 1998; (9) Aufdenberg et al. 2006; (10) Dyck et al. 1996; (11) CHARM2 catalog (Richichi et al. 2005).

applications of the data pipeline can be found in Monnier et al. (2002) and Tuthill et al. (2002). In principle, the pupil geometry of the telescope mimics the operation of a separate-element interferometer array, and the data collection and analysis are similar to standard methods for interferometry experiments such as speckle imaging. Masking interferometry generates complex visibilities (visibilities and closure phases) as its prime observables. For most objects in this study, the closure phases were small due to the baselines not sampling the visibility curve beyond the first null. For W Hya and $o$ Cet small (but significant) closure phases were measured indicating noncentrosymmetric brightness distribution, but discussion of these will be deferred to a following paper. Asymmetries have been reported for all Mira variables in our study except R Hya (see Scholz 2003).

The short-exposure images are dark-subtracted, flat-fielded, and cleaned of pattern noise. Power spectra are then computed frame by frame as the squared modulus of the Fourier transform. Stellar fringes appear as discrete peaks in such power spectra, with the origin occupied by a peak whose height is proportional to the squared flux in the frame. Squared visibilities are found by dividing the power at the spatial frequency of the fringes by that at the origin and then normalizing with the corresponding signal from the calibrator spectrum. The uncertainty associated with the squared visibilities is derived from the scatter in each ensemble of 100 exposures.

\subsection{Seeing Correction}

For all baselines and for all target and calibrator stars considered in this paper, the dominant noise sources were seeing and wind shake (wind-induced telescope wobble). When the atmospheric conditions vary between observing the source and its calibrator, the overall ratio changes between the fringe power and the total flux on the detector. As clarified by Monnier et al. (2004) for aperture-masking data, this change is nearly constant as a function of baseline for baselines longer than the coherence length ( $\approx 0.5 \mathrm{~m}$ at $K 2.26$ band). This means that the visibility function will approach a nonunity value at short baselines. We 
TABLE 4

Observations of $o$ Cet

\begin{tabular}{ccccccc}
\hline \hline Date & $\begin{array}{c}\mathrm{JD} \\
(-2,450,000)\end{array}$ & $\Phi$ & $\begin{array}{c}\mathrm{UD}_{J 1.24} \\
(\mathrm{mas})\end{array}$ & $\begin{array}{c}\mathrm{UD}_{H 1.65} \\
(\mathrm{mas})\end{array}$ & $\begin{array}{c}\mathrm{UD}_{K 2.26} \\
(\mathrm{mas})\end{array}$ & $\begin{array}{c}\mathrm{UD}_{L 3.08} \\
\text { (mas) }\end{array}$ \\
\hline 1997 Dec 16..... & 800 & 0.94 & $22.2 \pm 1.0$ & $27.5_{-2.2}^{+2.4}$ & $31.0 \pm 1.9$ & $58.8 \pm 1.5$ \\
Calibrators
\end{tabular}

TABLE 5

Observations of R Leo

\begin{tabular}{|c|c|c|c|c|c|c|c|}
\hline Date & $\begin{array}{c}\text { JD } \\
(-2,450,000)\end{array}$ & $\Phi$ & $\begin{array}{l}\mathrm{UD}_{J} 1.24 \\
\text { (mas) }\end{array}$ & $\begin{array}{l}\mathrm{UD}_{H} 1.65 \\
\text { (mas) }\end{array}$ & $\begin{array}{l}\mathrm{UD}_{K} 2.26 \\
(\mathrm{mas})\end{array}$ & $\begin{array}{l}\mathrm{UD}_{L 3.08} \\
\text { (mas) }\end{array}$ & Calibrators \\
\hline 1997 Jan $29 . \ldots \ldots . .$. & 478 & 0.05 & $\begin{aligned} 31.0 & \pm 1.1 \\
32.6 & \pm 1.5\end{aligned}$ & $29.6 \pm 1.9$ & $30.3 \pm 2.6,32.9_{-2.2}^{+2.3}$ & 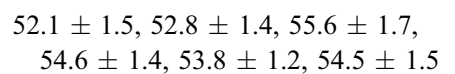 & $\alpha$ Hya, $\pi$ Leo \\
\hline 1997 Dec $16 \ldots \ldots$. & 800 & 1.14 & $\begin{aligned} 30.0 & \pm 1.1 \\
29.9 & \pm 1.2\end{aligned}$ & $\ldots$ & $31.4 \pm 1.7$ & $47.8 \pm 1.8$ & $\alpha$ Hya \\
\hline 1998 Apr $14 \ldots \ldots$. & 918 & 1.54 & $\ldots$ & $32.9 \pm 1.4$ & $32.3 \pm 1.8$ & $49.2 \pm 1.6$ & $\alpha$ Lyn \\
\hline 1998 Jun 4 .......... & 970 & 1.71 & $33.1 \pm 2.0$ & $29.7 \pm 2.0$ & $32.6 \pm 1.9$ & $50.7 \pm 1.6$ & $\alpha$ Lyn \\
\hline 1999 Apr $25 \ldots \ldots .$. & 1295 & 2.75 & $29.6 \pm 1.4$ & $29.1 \pm 0.8$ & $34.6 \pm 1.3,34.1 \pm 1.4$ & $53.3 \pm 1.5$ & $\alpha$ Lyn, $\pi$ Leo \\
\hline 2000 Jan $25 \ldots \ldots \ldots$ & 1570 & 3.64 & $\ldots$ & $34.6 \pm 1.6$ & $33.1 \pm 1.7$ & $52.6 \pm 0.7,52.1 \pm 0.8$ & $\alpha \operatorname{Lyn}$ \\
\hline 2000 Jun $23 \ldots \ldots$. & 1720 & 4.12 & $\ldots$ & $29.3 \pm 2.1$ & $31.2 \pm 1.9$ & $55.4 \pm 1.8$ & $\pi$ Leo \\
\hline 2001 Jun 11 ........ & 2073 & 5.24 & $31.5 \pm 1.3$ & $33.5_{-3.8}^{+4.6}$ & $30.7_{-3.8}^{+4.5}$ & $56.5 \pm 2.0$ & $\pi$ Leo, $\alpha$ Lyn, 2 Cen \\
\hline 2003 May $12 \ldots \ldots$. & 2772 & 7.48 & $\ldots$ & $36.2 \pm 1.1$ & $37.1_{-4.4}^{+5.8}$ & $\ldots$ & $\pi$ Leo \\
\hline 2004 May $28 . . . . .$. & 3154 & 8.71 & $\ldots$ & $30.1_{-1.8}^{+2.0}$ & $32.1 \pm 1.7$ & $56.4 \pm 1.4$ & $\pi$ Leo \\
\hline
\end{tabular}

TABLE 6

Observations of R Cas

\begin{tabular}{|c|c|c|c|c|c|c|c|}
\hline Date & $\begin{array}{c}\text { JD } \\
(-2,450,000)\end{array}$ & $\Phi$ & $\begin{array}{l}\mathrm{UD}_{J 1.24} \\
(\mathrm{mas})\end{array}$ & $\begin{array}{l}\mathrm{UD}_{H 1.65} \\
\quad(\mathrm{mas})\end{array}$ & $\begin{array}{l}\mathrm{UD}_{K} 2.26 \\
\quad(\mathrm{mas})\end{array}$ & $\begin{array}{l}\mathrm{UD}_{L 3.08} \\
\text { (mas) }\end{array}$ & Calibrators \\
\hline 1997 Dec 8 .......... & 802 & 0.67 & $\ldots$ & $\cdots$ & $30.0 \pm 1.5$ & $\ldots$ & $\alpha$ Cas \\
\hline 1998 Jun 4 ............ & 970 & 1.06 & $25.0 \pm 1.2$ & $23.2_{-2.7}^{+3.1}$ & $24.2 \pm 1.6$ & $44.8 \pm 2.2$ & $\gamma$ Sge, $\alpha$ Cas \\
\hline 1998 Sep $29 \ldots \ldots \ldots$ & 1056 & 1.27 & $24.5 \pm 1.5$ & $27.0_{-2.2}^{+2.4}$ & $\ldots$ & $41.1 \pm 3.0$ & $\alpha$ Cas \\
\hline 1999 Jan $5 \ldots \ldots \ldots \ldots$ & 1184 & 1.57 & $24.4 \pm 1.8$ & $23.9_{-2.4}^{+2.8}$ & $28.2 \pm 1.2$ & $44.1 \pm 1.8$ & $\alpha$ Cas \\
\hline 1999 Jul 29 .......... & 1390 & 2.04 & $24.1 \pm 1.8$ & $22.4_{-3.0}^{+3.4}$ & $28.5 \pm 1.4$ & $48.3 \pm 1.5$ & $\alpha$ Cas \\
\hline 2000 Jan $25 \ldots \ldots \ldots$ & 1570 & 2.46 & $\ldots$ & $27.7_{-2.0}^{+2.2}$ & $29.7 \pm 1.2$ & $\ldots$ & $\alpha$ Cas \\
\hline 2000 Jun $23 \ldots \ldots \ldots$ & 1720 & 2.81 & $24.9 \pm 1.4$ & $26.1_{-2.3}^{+2.6}$ & $28.9 \pm 1.9$ & $46.2 \pm 1.6$ & $\alpha$ Cas \\
\hline 2001 Jun $11 \ldots \ldots \ldots$ & 2073 & 3.63 & $\ldots$ & $26.5_{-2.2}^{+2.4}$ & $27.4_{-4.5}^{+5.4}, 27.6_{-4.5}^{+5.4}, 27.7_{-4.3}^{+5.2}$ & $\ldots$ & $\alpha$ Cas \\
\hline 2001 Jul 29 .......... & 2121 & 3.74 & $\ldots$ & $25.5_{-2.4}^{+2.7}$ & $26.1 \pm 2.1$ & $\ldots$ & $\alpha$ Cas \\
\hline 2002 Jul 23 .......... & 2479 & 4.58 & $\ldots$ & $29.5 \pm 0.9,27.4 \pm 0.9$ & $29.6 \pm 1.5$ & $42.0 \pm 2.0$ & $\alpha$ Cas \\
\hline 2003 May $12 . . . \ldots . .$. & 2772 & 5.26 & $\cdots$ & $23.3 \pm 2.3,24.4 \pm 1.4$ & $22.1_{-7.7}^{+14.3}, 29.0_{-6.0}^{+8.0}$ & $\ldots$ & $\delta \mathrm{Sgr}$ \\
\hline 2004 May $28 \ldots \ldots . .$. & 3154 & 6.14 & $\ldots$ & $23.2_{-2.5}^{+2.9}$ & $24.6_{-2.0}^{+2.2}$ & $48.9 \pm 1.5$ & 4 Cas \\
\hline 2004 Sep $2 \ldots \ldots \ldots \ldots$ & 3262 & 6.39 & $\begin{aligned} 27.6 & \pm 0.9 \\
26.6 & \pm 1.1\end{aligned}$ & $\begin{array}{c}26.8_{-1.6}^{+1.8}, 26.5 \pm 1.8 \\
27.9 \pm 2.0\end{array}$ & $25.2 \pm 1.5,24.5 \pm 1.3$ & $42.2 \pm 1.3,40.5 \pm 2.1$ & $\beta$ Cet, $\alpha$ Cas, $\epsilon$ Cyg \\
\hline
\end{tabular}




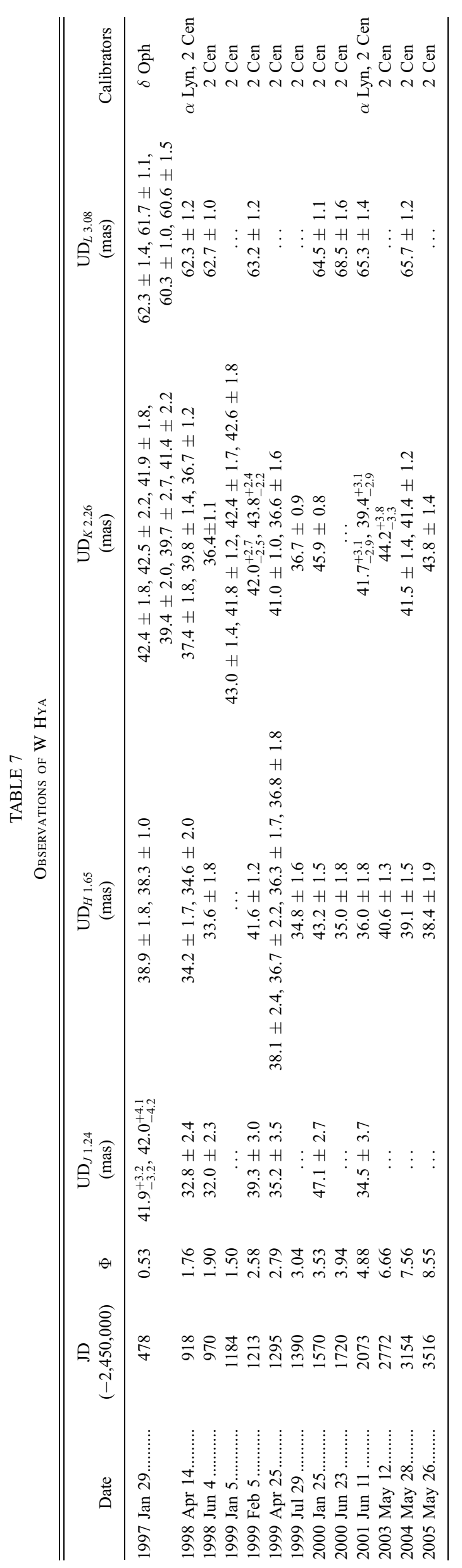


TABLE 8

Observations of $\chi$ Cyg

\begin{tabular}{|c|c|c|c|c|c|c|c|}
\hline Date & $\begin{array}{c}\text { JD } \\
(-2,450,000)\end{array}$ & $\Phi$ & $\begin{array}{l}\mathrm{UD}_{J 1.24} \\
\text { (mas) }\end{array}$ & $\begin{array}{l}\mathrm{UD}_{H 1.65} \\
\text { (mas) }\end{array}$ & $\begin{array}{l}\mathrm{UD}_{K} 2.26 \\
\text { (mas) }\end{array}$ & $\begin{array}{c}\mathrm{UD}_{L 3.08} \\
\text { (mas) }\end{array}$ & Calibrators \\
\hline 1998 Jun 4 .............. & 9700 & 0.59 & $21.3 \pm 2.8$ & $19.5_{-3.1}^{+3.9}$ & $23.0 \pm 2.1$ & $45.6 \pm 3.0$ & $\gamma$ Sge \\
\hline 1998 Sep $29 . \ldots \ldots \ldots \ldots$. & 1056 & 0.87 & $\ldots$ & $21.2_{-2.9}^{+3.4}$ & $26.1 \pm 2.7$ & $46.0 \pm 4.0$ & $\gamma$ Sge \\
\hline 1999 Jul 29 ............. & 1390 & 1.62 & $21.6 \pm 1.6$ & $22.4_{-2.7}^{+3.2}$ & $28.2 \pm 1.5$ & $42.4 \pm 3.0,43.7 \pm 2.8$ & $\gamma$ Sge, Vega \\
\hline 2000 Jun $23 \ldots \ldots \ldots \ldots$. & 1720 & 2.48 & $\ldots$ & $23.1_{-2.6}^{+3.0}$ & $25.1 \pm 2.2,24.9_{-2.1}^{+2.3}$ & $39.5 \pm 2.4,41.0 \pm 2.7$ & $\gamma$ Sge, $\xi$ Cyg \\
\hline 2001 Jun 11 ............. & 2073 & 3.36 & $25.4 \pm 1.9$ & $26.4_{-2.5}^{+2.8}$ & $30.4_{-4.1}^{+4.7}$ & $45.5 \pm 2.5$ & $\gamma$ Sge \\
\hline 2002 Jul 23 ............ & 2479 & 4.36 & $28.5 \pm 2.7$ & $27.5 \pm 1.0$ & $29.6 \pm 1.6$ & $44.3 \pm 2.5$ & $\gamma$ Sge \\
\hline 2003 May $12 \ldots \ldots \ldots . .$. & 2772 & 5.07 & $\ldots$ & $28.1 \pm 2.0$ & $42.1_{-3.9}^{+4.4}$ & $46.5 \pm 2.6$ & $\epsilon \mathrm{Cyg}$ \\
\hline 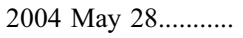 & 3154 & 6.01 & $\ldots$ & $24.7_{-2.7}^{+3.0}$ & $33.8 \pm 3.5$ & $53.1 \pm 4.0$ & Vega \\
\hline 2004 Sep $23 \ldots \ldots \ldots \ldots . .$. & 3262 & 6.31 & $24.8 \pm 1.2$ & $26.7 \pm 2.0$ & $33.0 \pm 2.3$ & $50.5 \pm 3.9$ & $\gamma$ Sge \\
\hline
\end{tabular}

took the visibility at the origin to be a free parameter in our studies, an assumption that does not affect our fitting procedures as long as there is no significant flux coming from overresolved structures $\left(>0.5^{\prime \prime}\right)$. Seeing and wind shake also lowered the mean visibility $V$ and increased its variance on each baseline. However, the quantity $\operatorname{Var}(V) /\left\langle V^{2}\right\rangle$ was independent of the stellar brightness and the degree to which it was resolved by the baseline in question, only depending on seeing and wind shake. We partially corrected for the effects of seeing and wind shake using the method described by Ireland (2006), which entails empirically fitting a function of the form $V=V_{0} \exp \left\{-k\left[\operatorname{Var}(V) /\left\langle V^{2}\right\rangle\right]\right\}$ to individual star observations and correcting visibilities for each target and calibrator star prior to dividing the target with calibrator visibilities. We used a conservative value for $k$ of 0.8 (which may have undercorrected for the effects of seeing and wind shake) and smoothed the function $\operatorname{Var}(V) /\left\langle V^{2}\right\rangle$ in the $u-v$ plane in order to minimize errors in applying this correction (for further details see Ireland 2006). We evaluated the effectiveness of this correction by comparing the calibrated visibilities of unresolved stars with and without seeing correction, which turned out to improve the calibration process. The apparent angular diameters were closer to the true values after the seeing/wind shake correction on $>70 \%$ of the stars examined (see Fig. 1). Only on three object observations with very bad wind shake did the algorithm cause a marginal worsening of the miscalibration (5 mas).

Figure 2 shows typical data obtained from the masking experiment. The targets were seldom fully resolved, making image reconstruction only possible for those objects with the largest angular diameters (e.g., W Hya). These results will be presented in a subsequent paper. The two-dimensional visibilities showed no significant deviation from circular symmetry (except for W Hya), possibly due to the lack of high spatial frequencies sampled by our $<10 \mathrm{~m}$ baselines. Since W Hya's deviation from circular symmetry is smaller than the uniform disk (UD) angular diameter error, we made the assumption that all the stars are spherical and the data were azimuthally averaged.
To keep the representation of the data homogeneous, our quantitative analysis is based on fitting simple UD models to the azimuthally averaged Fourier data. We concentrate on the four filters that provide most of the temporal coverage in our observations, namely, $L 3.08, K 2.26, H 1.65$, and $J 1.24$, considering the other filters only in $\S 4$.1. Here, e.g., $L 3.08$ indicates that the filter covers a subinterval of the $L$ bandpass of the conventional $U B V$ filter system and is centered at $3.08 \mu \mathrm{m}$ (Table 2). Note that the $K 2.26$ bandpass encompasses two very similar filters, both centered at $2.26 \mu \mathrm{m}$ with slightly different bandwidths.

Calibrator stars nearby in the sky and measured interleaved with the target observation are used to estimate the system transfer function, a standard practice in interferometry described in detail by Millan-Gabet et al. (2005).

The visibility amplitude was calibrated by dividing the target visibilities by the calibrator star visibilities, after first correcting for the estimated sizes of the calibrators (Table 3 ).

\subsection{Uniform Disk Diameters}

Although the true stellar intensity profile is not a UD, fitting the observed visibilities with this simple profile still provides a useful estimate of the apparent size of the target. Our longest baseline was just under $10 \mathrm{~m}$, and so we are resolving low-resolution structure in the target star's intensity profile. This makes it difficult to differentiate between a UD, a fully darkened disk, or a Gaussian. Since the real intensity distribution is none of these simple models, and converting a Gaussian or a more complex intensity distribution into a radius is rather arbitrary, we chose UD diameters to allow comparison of findings with existing literature and to avoid the difficulties encountered when deriving more sophisticated diameters (cf. Hofmann et al. 1998; Scholz 2003). If the "true" intensity distribution is known, or a predicted model distribution is to be compared with the data, different radius definitions can be easily converted from and to a UD radius. In the cases of $o$ Cet and W Hya, the $J 1.24$ band curves show some deviation from the UD model at around $3 \times 10^{6} \mathrm{rad}^{-1}$, which could indicate the

TABLE 9

ObSeRvations of R Hya

\begin{tabular}{|c|c|c|c|c|c|c|c|}
\hline Date & $\begin{array}{c}\mathrm{JD} \\
(-2,450,000)\end{array}$ & $\Phi$ & $\begin{array}{l}\mathrm{UD}_{J 1.24} \\
\text { (mas) }\end{array}$ & $\begin{array}{c}\mathrm{UD}_{H} 1.65 \\
\text { (mas) }\end{array}$ & $\begin{array}{c}\mathrm{UD}_{K 2.26} \\
\text { (mas) }\end{array}$ & $\begin{array}{c}\mathrm{UD}_{L 3.08} \\
\text { (mas) }\end{array}$ & Calibrators \\
\hline 1997 Jan $29 \ldots \ldots \ldots \ldots$ & 478 & 0.70 & $26.2 \pm 1.9$ & $\ldots$ & $26.0_{-2.5}^{+2.8}$ & $40.0 \pm 2.1$ & $\delta \mathrm{Oph}$ \\
\hline 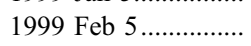 & 1213 & 2.64 & $\ldots$ & $30.0_{-2.0}^{+2.2}$ & $\ldots$ & $\ldots$ & $\pi$ Hya \\
\hline 1999 Apr $25 \ldots \ldots \ldots \ldots$ & 1295 & 2.86 & $\ldots$ & $24.1 \pm 2.9$ & $28.0 \pm 2.2$ & $\ldots$ & 2 Cen \\
\hline 2000 Jan $25 \ldots \ldots \ldots \ldots . . . .$. & 1570 & 3.58 & $\ldots$ & $\ldots$ & $31.0 \pm 1.3$ & $\ldots$ & 2 Cen \\
\hline 2003 May $28 \ldots \ldots \ldots \ldots . . .$. & 2772 & 6.74 & $\ldots$ & $\ldots$ & $27.2_{-6.3}^{+9.0}$ & $\ldots$ & 2 Cen \\
\hline
\end{tabular}



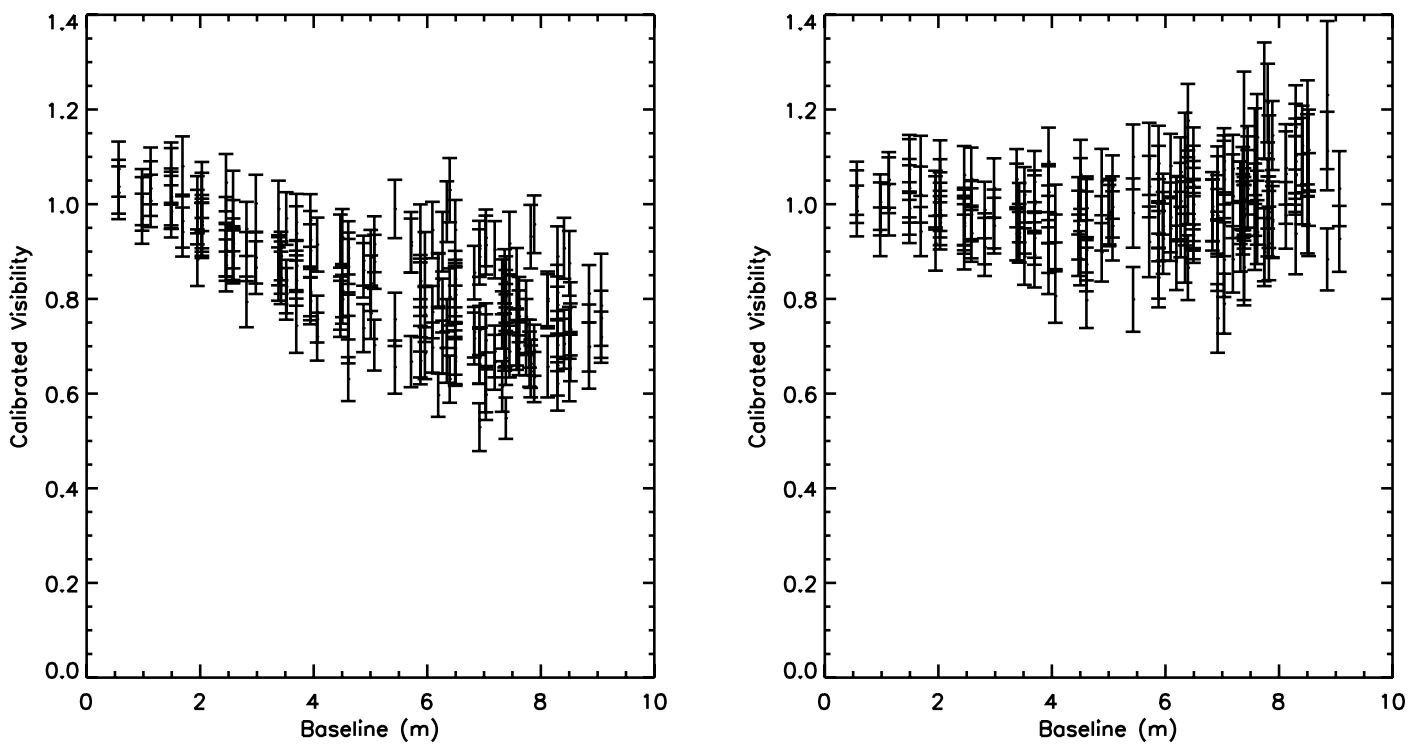

FIG. 1.-Left: Calibrated visibilities (without azimuthal averaging) of an unresolved target star ( $\alpha$ Ari). Due to the effects of variable seeing and/or wind shake, the object appears to have a UD angular diameter of 23 mas. Right: Same data with the seeing correction applied, showing that these data are now consistent with an unresolved source.

presence of dust causing scattering in the outer layers, leading to a smaller true photospheric diameter (see Fig. 2) than derived with a simple UD model fit. In addition, due to their larger angular diameters (especially in the $L 3.08$ filter), W Hya and possibly $o$ Cet were also resolved enough to detect further deviations of the intensity distribution from the simple UD shape to be compared with model predictions. They are also possible targets for imaging after recovering the closure phase information in addition to the Fourier amplitudes, whereas the other Mira variables in our sample do not yield useful closure phase information. These comparisons with more complex models and interpretations will be addressed in a future paper.

\subsection{Estimation of Seeing Miscalibration}

Given that the seeing correction described in $\S 2.3$ leaves some residual error and assuming that the dominant error term is the uncertainty introduced by variations in the seeing between measurements of targets and calibrators, we undertook a separate study that assessed the robustness and repeatability of the calibration process to seeing-induced miscalibration. If the seeing remains constant, then calibrating (i.e., dividing) the visibility function from successive data sets taken on the same object should yield the visibility function of a point source (i.e., $V^{2}=1$ ). In the case of changing atmospheric conditions, considerable differences in the transfer function can appear between consecutive data sets. The error induced by such fluctuations can be measured from observations of a single object (either target or calibrator) taken through the same filter at different times during one night. Once again, we chose to fit a UD model to the data, a natural candidate to fit the error introduced by the change in seeing throughout one observing night. This allows us to estimate atmospheric uncertainties by applying the same fitting procedure as was used for the rest of our data, reducing the risk of biases introduced by the fitting method.

Asymmetric errors were calculated by calibrating the target with a UD of the derived diameter. These errors pertain to the respective filters, and if no object was observed twice in one night in the same band, the maximum global error is assumed.
The data in Figure 2 demonstrate another well-known problem: that of calibration near the so-called seeing spike. Note how the azimuthally averaged visibility points at short baselines can deviate considerably from the otherwise uniform shape of the visibility curve. As the calibration of these large fluctuations at low spatial frequencies is highly challenging, we fitted our models only to the spatial frequencies in Fourier space corresponding to baselines of $2 \mathrm{~m}$ or more.

\subsection{NIR Photometry}

In the course of the data reduction, we extracted the total received flux for both science objects and calibrators. With this we were able to retrieve contemporaneous photometry data in the same bands as our angular diameter measurements. These measurements complement the photometry by Whitelock et al. (2000), which does not cover our full range of observations, and provide a powerful tool to monitor NIR light curves. The small differences between the Whitelock et al. (2000) photometry and our measurements can be attributed to our use of filters of differing bandpass and center wavelength (see Table 2).

\section{RESULTS}

\subsection{Light Curves}

Figure 3 shows our NIR photometry plotted as a function of pulsation phase, together with the $J H K$ photometry of Whitelock et al. (2000) and AAVSO visual photometry (A. A. Henden et al. 2006, private communication). As an approximation based on the appearance of the light curves, we fitted our NIR photometry data with simple sinusoidal functions (setting the frequency to the known period and using amplitude, phase, and average value as free parameters, not shown in the figures), with the exception of R Hya, where the light curve was too incomplete. Although these fits are only approximations, they provide a useful tool to examine the dates of maxima and minima as a function of wavelength. The NIR magnitude versus phase curves are certainly not strictly sinusoidal, but depending on the star and the bandpass they can be approximated by a sine function. Our photometry shows a 

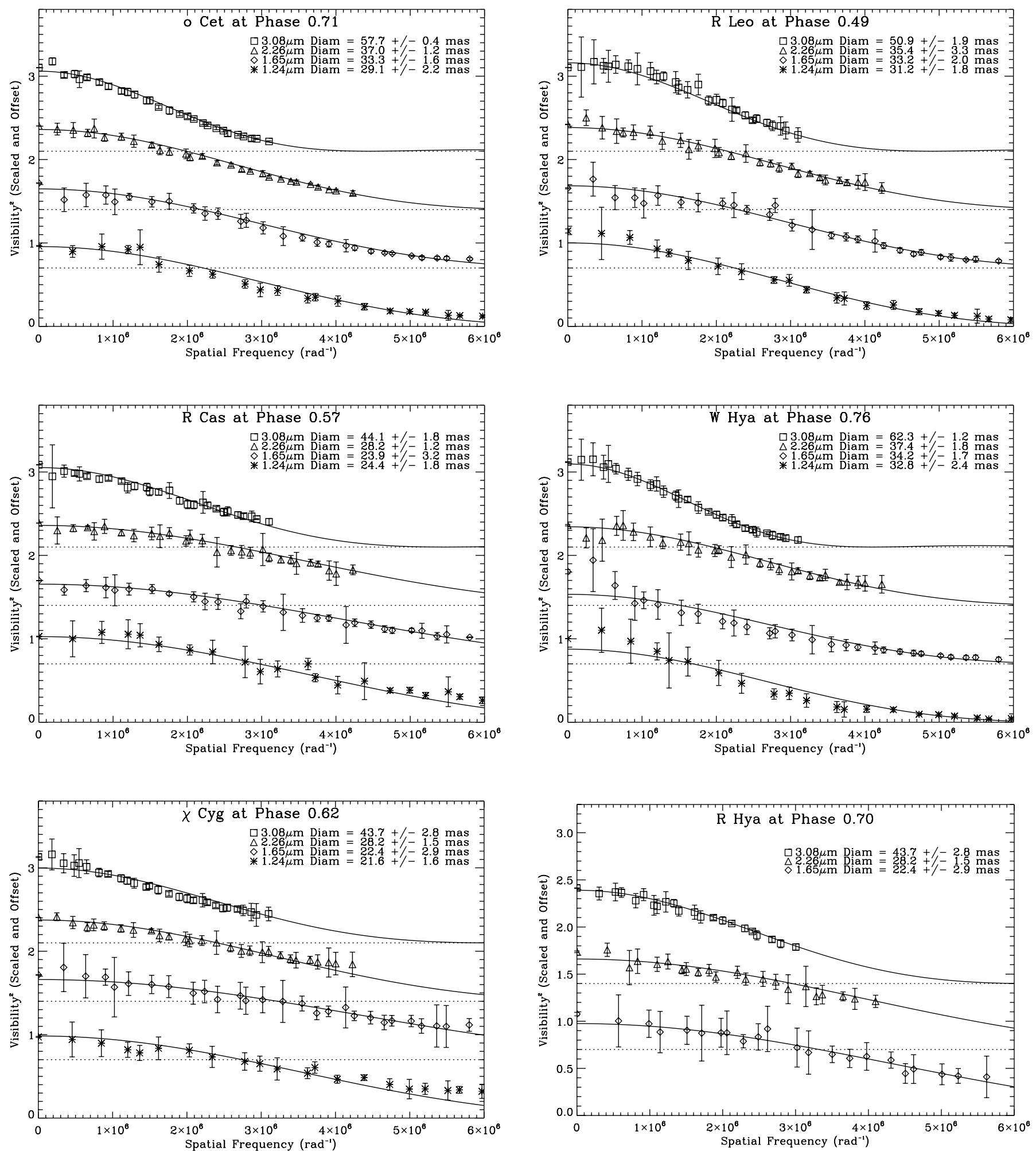

FIG. 2.-Azimuthally averaged squared visibility functions for $o$ Cet, R Leo, R Cas, W Hya, $\chi$ Cyg, and R Hya at various visual phases. Results for the different filters are offset for clarity. The very large apparent diameters in the $L 3.08$ filter can be attributed mainly to the $\mathrm{H}_{2} \mathrm{O}$ opacity, as first suggested and observed by Tuthill et al. (2000a). The diameters correspond to best-fit UD models.

small lag of up to 0.07 cycles in the maxima of light curves from the $J 1.24$ to the $H 1.65$ and from the $H 1.65$ to the $K 2.26$ filters, as well as less pronounced or nonexisting lags near visual minimum, confirming the findings of Smith et al. (2002) as can be seen in Figure 3. The reported phase shift of $\approx 0.15-0.22$ with which the NIR maxima lag behind visual maxima typical for M-type Mira variables (Nadzhip et al. 2001; Smith et al. 2002) is also clearly seen in our data. Also in accordance with the findings of Smith et al. (2002), we find that the maxima in longer wavelengths (i.e., in the $L$ band) occur before the $K$ maxima but after the visual maxima for all of our objects except for W Hya, where the $L$ light curve is too peculiar to be fitted with a sine function. 

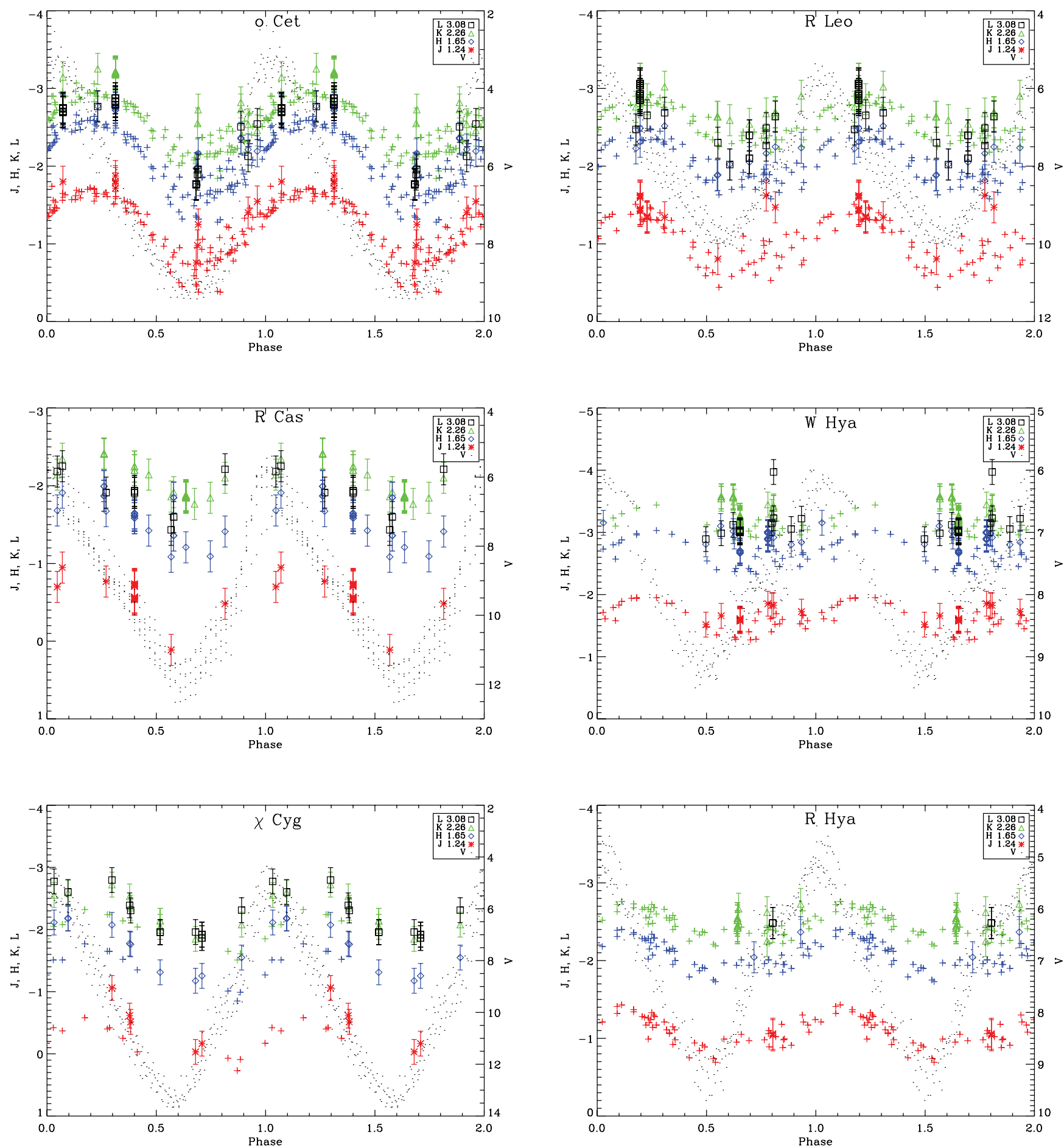

FIG. 3.-Our NIR photometry folded with the visual pulsation period, shown with Whitelock et al. (2000) JHK photometry and visual light curves. The $J 1.24$, $H 1.65, K 2.26$, and $L 3.08$ fluxes extracted from NIRC masking are represented by red asterisks, blue diamonds, green triangles, and black squares, respectively. Whitelock et al. (2000) J (red plus signs), $H$ (blue plus signs), and $K$ photometry data (green plus signs) are shown where available. The dots show the visual magnitude from the AAVSO database averaged into 10 day bins (A.A. Henden et al. 2006, private communication), taken during the period of our observations.

Both Visible and NIR light curves given in Figure 3 span the observation period of each object and clearly illustrate the substantial differences between the cycle amplitudes and pulsation periods.

\subsection{Multiwavelength, Multiepoch UD Angular Diameters}

Figures 4-6 show the UD angular diameters of the sample of Mira variables at various visual phases (see Tables 4-9), folded with phase, together with visual photometry data from the AAVSO (A. A. Henden et al. 2006, private communication). We have detected diameter variations for all Mira variables in this study and compared these results with the NIR pulsations of Mira stars that have been observed interferometrically before (e.g., by Tuthill et al. 1995; Perrin et al. 1999; Young et al. 2000; Thompson et al. 2002; Woodruff et al. 2004; Fedele et al. 2005; Ragland et al. 2006). As discussed for the light curves in $\S 3.1$, the physical 

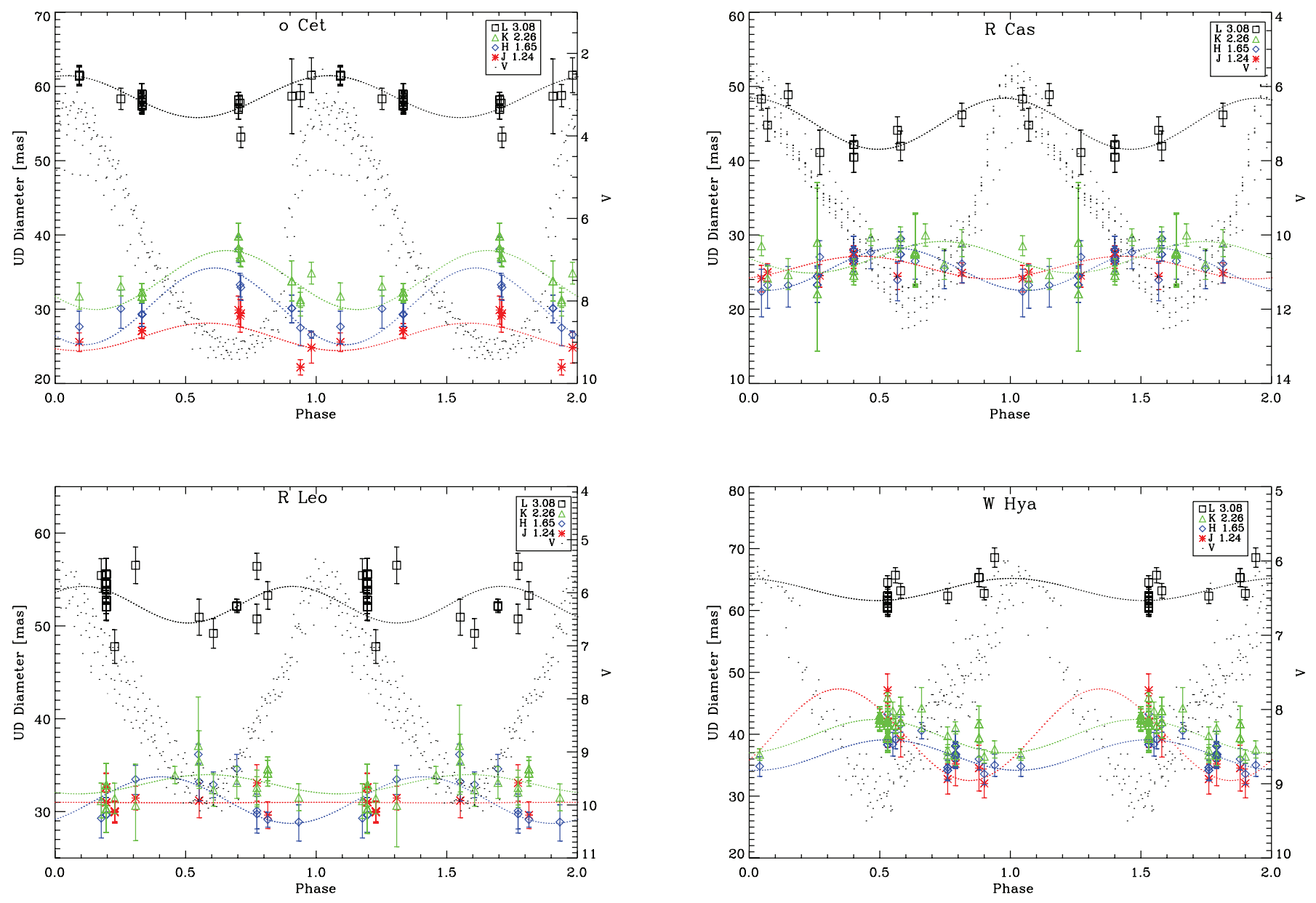

FIG. 4.-UD angular diameters for $o$ Cet (top) and R Leo (bottom), folded with pulsation phase. The symbols with error bars show UD diameters for the $J 1.24$, $H 1.65, K 2.26$, and $L 3.08$ filters, and the dotted lines show the best-fitting sinusoids. The dots show the visual magnitude from the AAVSO database averaged into 10 day bins (A. A. Henden et al. 2006, private communication), taken during the period of our observations. Data have been replicated for two cycles to clarify the sinusoidal pulsation.

diameter versus phase curves are certainly not strictly sinusoidal. Nonetheless, a sinusoidal shape of the curve can be observed, and although not perfect, the sine curves fitted to the data have been included to guide the eye. With the extensive coverage of phases and cycles in four filter bandpasses, it becomes possible to probe the stellar atmosphere both for geometric pulsation of the continuum-forming layers (the so-called photosphere) and for contamination of the continuum by molecular blanketing. This allows us to further constrain existing theoretical models and to make more sophisticated demands on future models. R Hya will be exempt from further discussion as the data sampling is too sparse (see Fig. 6, bottom panel).

\section{DISCUSSION}

\subsection{Effects of Molecular Absorption on Multiwavelength Diameter Observations}

Figure 7 shows the NIR spectrum of a Mira (R Cha) at two different pulsation phases, with the position and shape of the $z 1.08$, $J 1.24, H 1.65$, and $K 2.26$ filters, and Figure 8 shows the Infrared Space Observatory (ISO) spectrum of R Cas, with the position and shape of the $L 3.08$ and $L 3.31$ filters. The narrowband filters $J 1.24$ and $H 1.65$ should show little molecular contamination by

absorption bands; i.e., they are close to sampling the continuumforming layers (see, e.g., Tej et al. 2003), whereas the contamination effect should be more prominent in the $K 2.26$ and in particular the $L$-band filters (see, e.g., Jacob \& Scholz 2002; Mennesson et al. 2002; Ireland et al. 2004a, 2004b). Within the $L$ band, our filters ( $L 3.08$ and $L 3.31$ ) sample portions with different molecular absorption lines, originating mainly from $\mathrm{H}_{2} \mathrm{O}$ (but also from $\mathrm{OH}$ and $\mathrm{SiO}$; Mennesson et al. 2002) in Mira atmospheres. The $L 3.08$ bandpass lies deeper in the absorption feature than the $L 3.31$ bandpass. The photons seen through the $L 3.08$ filter should therefore originate from cooler strata that lie higher in the stellar atmosphere than the $L 3.31$ photons.

Figure 9 shows the diameter versus wavelength relationship for $o$ Cet at four different phases. It clearly shows the effect of varying absorption features on the perceived diameter. As our filters coincidentally sample more opaque (contaminated) layers with increasing wavelength, there is a perceived diameter increase as a function of wavelength, with the exception of the $J 1.24$ and $L 3.31$ filters, which lie farther out of the molecular absorption bands sampled by the $z 1.08$ and $L 3.08$ filters, respectively. This increase of diameter with wavelength is obvious throughout the pulsation cycle for $o$ Cet, all other Mira variables showing excursions from the $J 1.24>H 1.65>K 2.26$ diameter trend at some phases. In particular, R Leo seems to show a much more complex layering (see $\S 4.3$ and Fig. 4). All stars in our sample show a large $(>30 \%)$ increase in UD diameter between the $K 2.26$ filter and the $L 3.08$ filter, first noted in the case of R Aqr by Tuthill et al. (2000a). This increase indicates that the $L 3.08$ filter samples a 

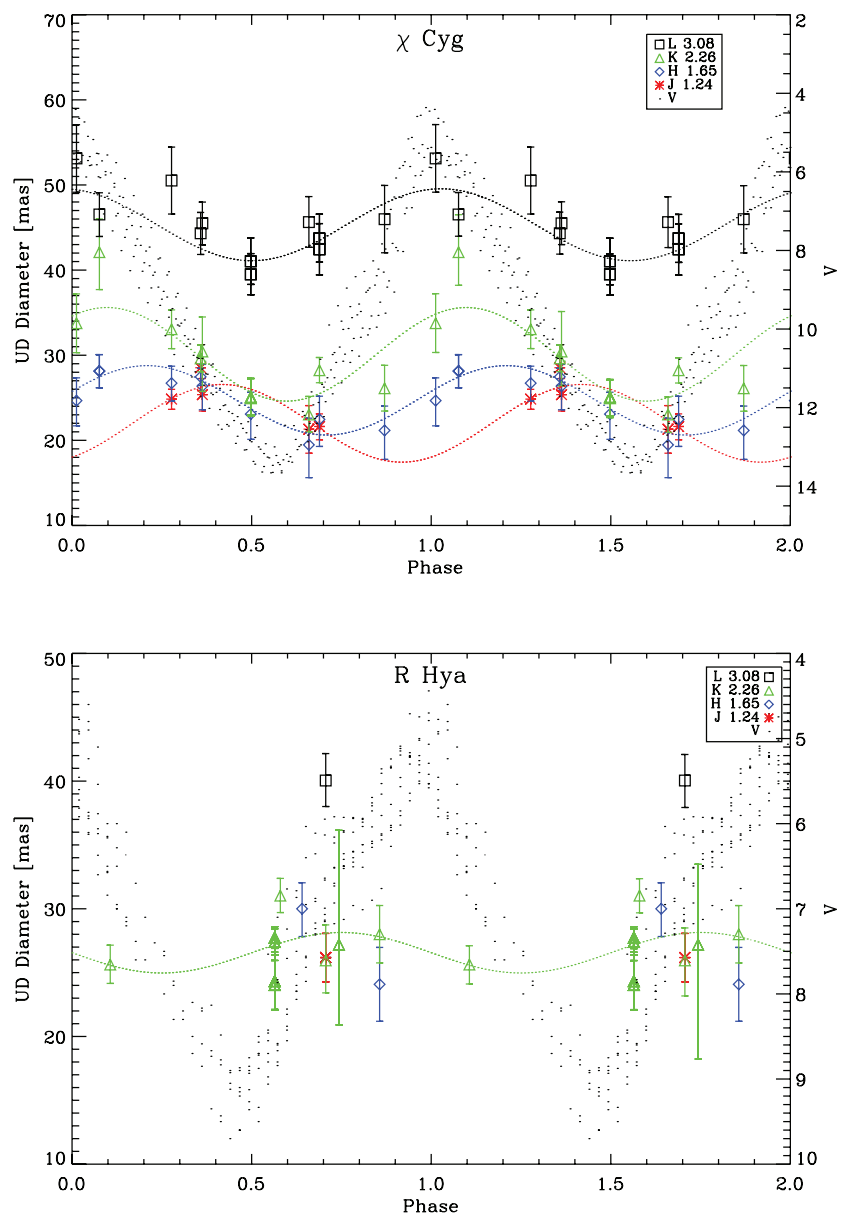

FIG. 6.- Same as Fig. 4, but for $\chi$ Cyg (top) and R Hya (bottom).

molecular layer at a considerable distance from the photosphere. The distance between the layers varies with phase and is further discussed below for each object individually, as it differs greatly from star to star.

\subsection{Phase Dependence of Multiwavelength NIR Diameters}

The distinction between true photospheric pulsation, i.e., the upward and downward motion of the continuum-forming layers, and the effects of molecular blanketing is not trivial to unravel, even with high angular resolution data. The varying molecular opacity is dictated by density changes as a shock front travels through the stellar atmosphere and temperature changes due to variations in the radiation field, causing molecules to dissociate and reform (cf. Scholz 2003 and references therein). These more or less opaque strata that vary with the pulsation phase (and between cycles) can make it difficult to derive the near-UD diameter of the underlying geometrically pulsating continuum layer, veiling the geometric amplitude and the phase of pulsation. As the brightness distributions of Mira variables are more complex than simple UDs, changes in molecular opacities of different layers may be capable of mimicking changes in our derived diameters, complicating the interpretation of our data.

Another layer of complexity is added by the possibility of material flow through the photosphere during a pulsation cycle, changing the angular size of higher atmospheric layers while having little influence on the photospheric angular diameter. Since the meaning of outflow and inflow velocities and of what is considered to be the photosphere requires careful model-based discussion, these points will be addressed in the subsequent model interpretation paper. The behavior of the S-type Mira $\chi$ Cyg differs from the other Mira variables in our sample in various respects and is discussed separately.

Of the three filters within the JHK bands, the atmospheric opacity reaches its highest values in the contaminated (albeit less contaminated than the standard $K$ bandpass) filter $K 2.26$ throughout most of the pulsation cycle, as evidenced in Figures $4-6$ by the large angular diameters. This increased molecular opacity has been predicted by various models (e.g., Jacob \& Scholz 2002; Ireland et al. 2004a, 2004b; Ireland \& Scholz 2006) and has been observed by, e.g., Millan-Gabet et al. (2005).

A diagnostic observable from our data that is more robust against sources of systematic errors (e.g., change in seeing, windinduced wobble of the telescope, calibrator characteristics) is the relative diameter variation. Figure 10 shows the relative UD diameter variation as a function of phase for the five M-type Mira variables in this study, averaged (variance weighted) into phase bins containing on average seven data points. The relative UD diameters were obtained by dividing each object's measured angular diameters by its mean, and the errors are representative of the scatter within each phase bin $(1 \sigma)$. The data illustrate the homogeneity within this sample of Mira variables and allow us to investigate systematic differences between the observed bandpasses. In order to extract phase offsets and relative pulsation amplitudes in each filter, we fitted sinusoid cycloids to the combined data, without implying that the objects vary in such a simple fashion.

The ensemble shows a systematic phase shift of the relative UD diameter variation with wavelength, not unlike the trend reported for the light curves in $\S 3.1$. The $J 1.24, H 1.65$, and $K 2.26 \mathrm{UD}$ diameters reach their minimum at approximately phases $0.9,1.0$, and 1.1, respectively. The $L 3.08$ bandpass diameters behave differently and are examined in more detail at the end of this section. This disagrees with theoretical model predictions by Ireland et al. (2004a, 2004b), where the diameter minimum should occur at roughly phases $0.7-0.8$ for all bandpasses, but is comparable to the findings of Thompson et al. (2002), who followed the M-type Mira S Lac through its pulsation cycle in various subfilters of the $K$ band. Using the sample as a single "artificial" Mira also allows us to verify the relative diameter pulsation amplitude for S Lac of Thompson et al. (2002), who report a $12 \%-21 \%$ peak-to-peak sinusoid amplitude in the $K$ band, with our value of $14 \%$ peakto-peak pulsation in the $K 2.26$ band. The peak-to-peak sinusoidal pulsation amplitudes for the $J 1.24, H 1.65$, and $L 3.08$ bandpasses are $14 \%, 22 \%$, and $6 \%$, respectively, also in disagreement with theoretical models that predict much higher amplitudes (see, e.g., Ireland et al. 2004a, 2004b).

According to current models (e.g., Ireland et al. 2004a, 2004b), more contaminated layers (such as the ones sampled by the $K 2.26$ and $L 3.08$ bandpasses) should experience slightly greater diameter pulsation amplitudes. This trend cannot be seen in our observations: the layer experiencing the greatest diameter pulsation is the less contaminated $H 1.65$ layer. Explanations for this could be as follows: (1) the relatively narrow width of our $K 2.26$ filter compared to the standard $K$ filter used in the models, combined with the position of our central wavelength in a possible minimum of molecular contamination as reported by Thompson et al. (2002); (2) a periodic change of optical depth of a layer, offset in phase to the photospheric pulsation, could result in large variations of the observed pulsation amplitude; (3) the assumed spherical symmetry could be significantly violated in the outer, more contaminated, layers (see Ragland et al. 2006 and references therein), effectively causing departures from model predictions (cf., e.g., Hofmann et al. 2000; Ireland et al. 2004c); (4) the theoretical models need revision to accommodate for these observations. 


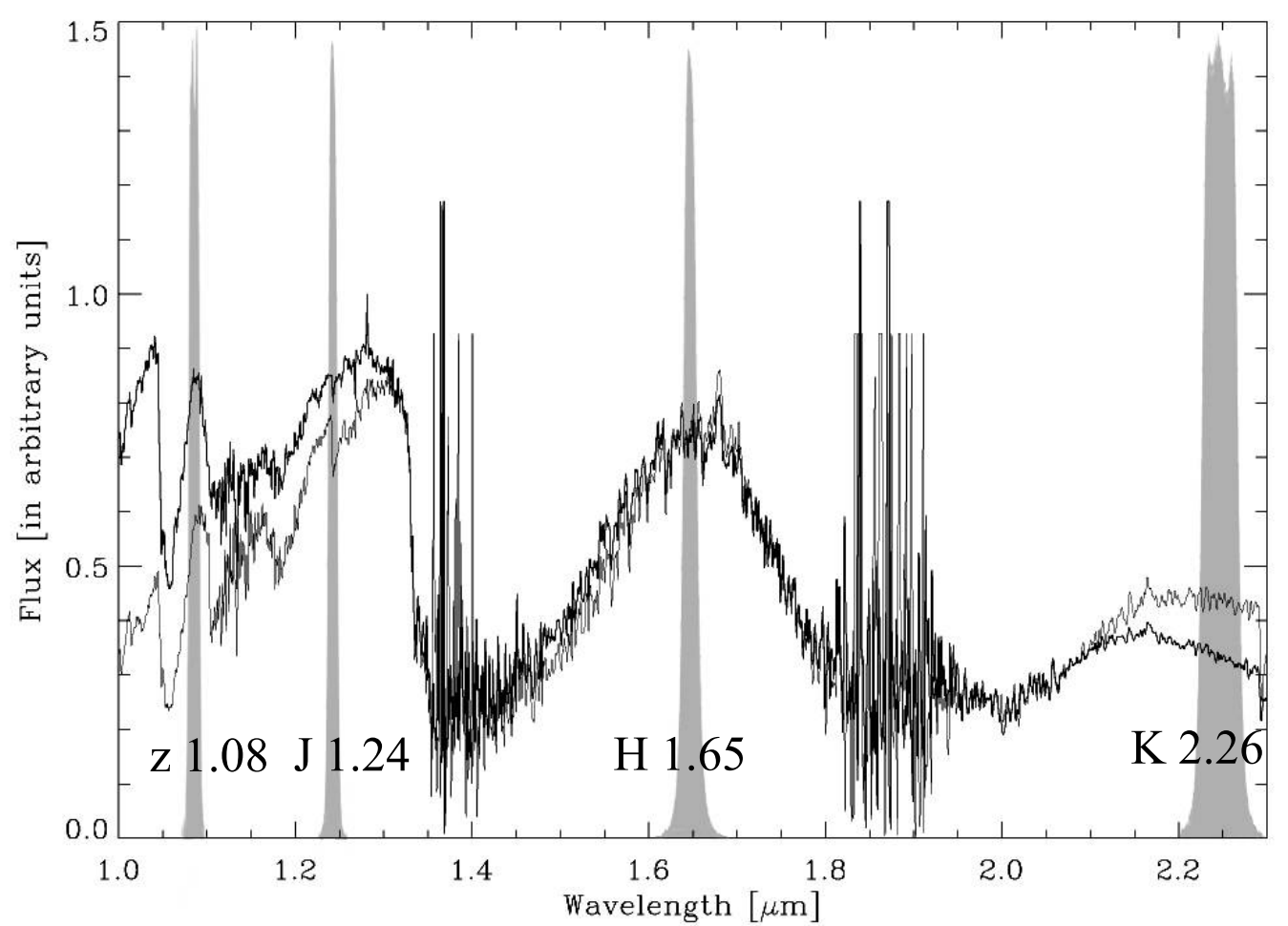

FIG. 7.-NIR spectrum of Mira R Cha at two different pulsation phases (1996 May 26 at phase 0.6 and 1996 March 3 at phase 0.3 ) from Lançon \& Wood (2000) showing the position and shape of the $z 1.08, J 1.24, H 1.65$, and $K 2.26$ filters. The $J 1.24$ and $H 1.65$ filters penetrate to layers that lie closer to the continuum-forming photosphere, whereas the $K 2.26$ filter sees a portion of the spectrum that is more contaminated by molecular opacities.

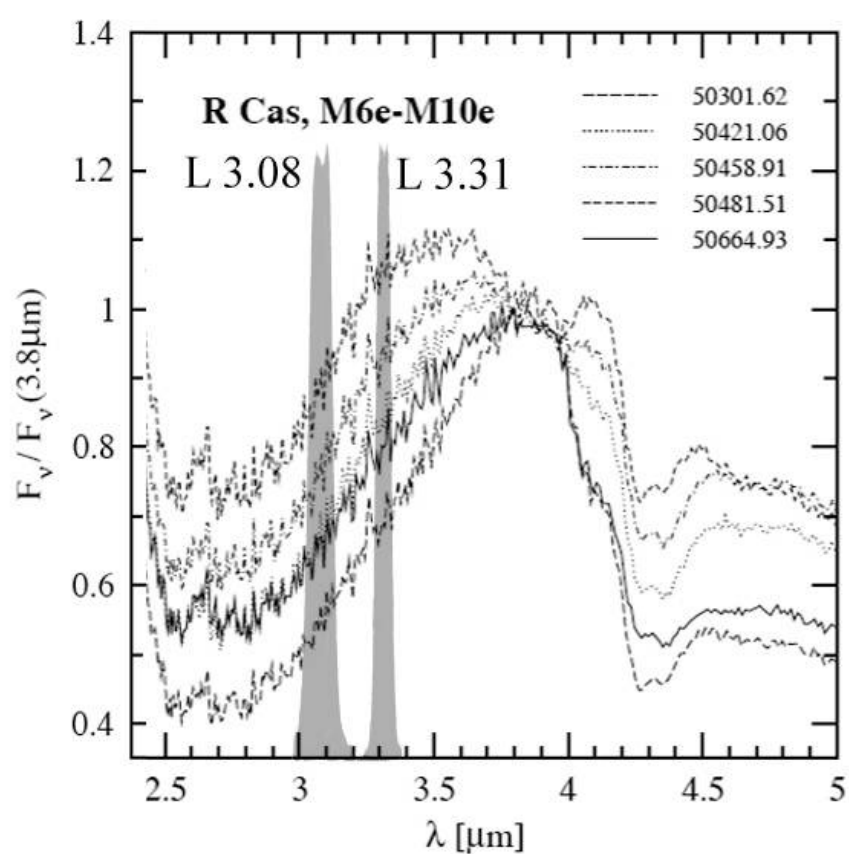

FIG. 8.- - Infrared ISO spectrum of R Cas at various phases, from Aringer et al. (2002, Fig. 2), with the position and shape of the $L 3.08$ and $L 3.31$ filters (Table 2) overlaid. The stronger absorption, mainly due to $\mathrm{H}_{2} \mathrm{O}$ molecules, in the $L 3.08$ filter comes from cooler strata (and thus farther away from the continuum-forming layers) and leads to the measurement of greater angular diameters. See text $(\S 4.1)$.
Higher molecular opacity can be expected at near-minimum phases, when the outer layers are cooler and more molecules are formed (see, e.g., Ireland et al. 2004a, 2004b). For our sample stars (with the exception of $\chi \mathrm{Cyg}$ ), the maximum apparent $J H K$ UD angular diameter values are typically found near minimum visual phase, supporting existing model interpretations.

A diagnostic observable from our data that is more robust against sources of systematic errors (e.g., change in seeing, windinduced wobble of the telescope, calibrator characteristics) are the relative diameter ratios. Figure 11 shows the UD diameter ratio between the different filters for all our sample stars plotted versus

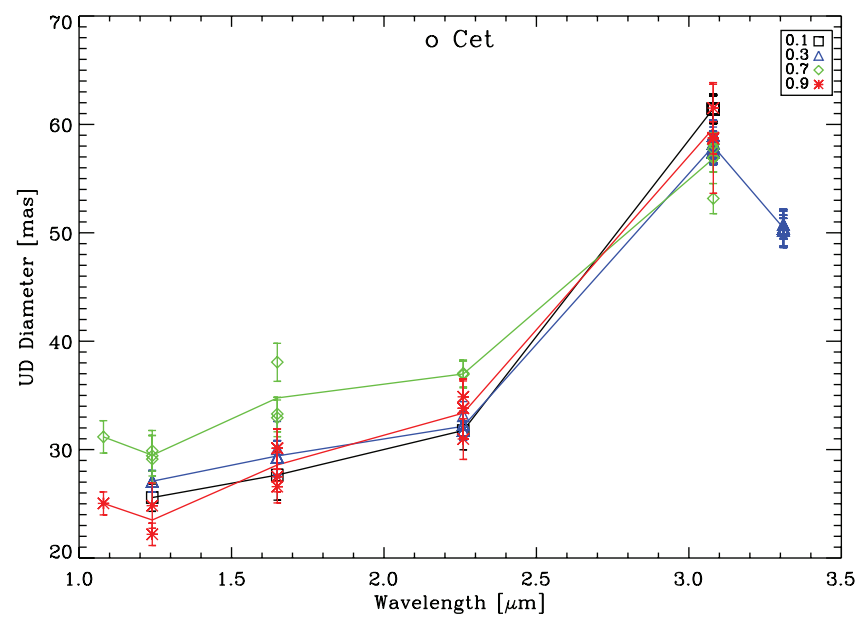

FIG. 9.-Diameter vs. wavelength relationship for $o$ Cet measured at visual phases $0.1,0.3,0.7$, and 0.9 . All visual phase measurements carry a binning error of \pm 0.1 . 


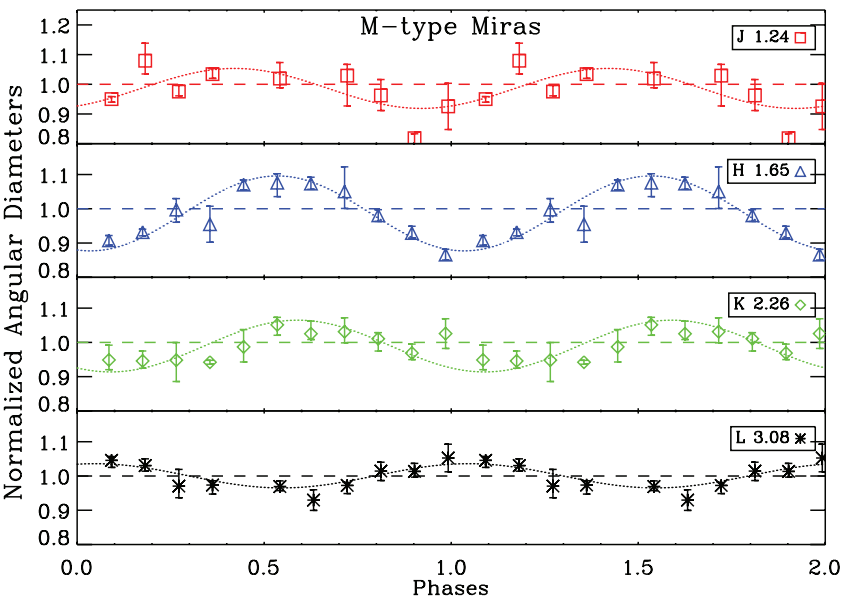

FIG. 10.- Relative angular diameter variation as a function of phase for the five M-type Mira variables in this study. The sine curves represent fits to the ensemble of angular diameters divided by the mean angular diameter for each star. The data have been averaged into phase bins (see text) and are repeated to show two cycles for better recognition of the pulsation pattern.

phase. There is no obvious dependence on pulsation phase of the $H 1.65 / J 1.24$ quotient, although there is noteworthy scatter for our sample of five Mira variables (R Hya was never observed simultaneously in both filters) that might mask a minor phase effect. The mean value of this diameter ratio is

$$
\overline{R_{H 1.65 / J 1.24}}=1.02 \pm 0.10,
$$

which agrees, to within errors, with the value of $\overline{R_{H / J}}=1.08 \pm$ 0.09 reported by Millan-Gabet et al. (2005). The lack of a phasedependent signature indicates a closeness in temperature and opacity variations, as can also be derived from the closeness in phase and pulsation amplitude seen in Figure 10. The diameter ratio close to unity shows the geometric closeness of the two layers.

Note that of all stars, $o$ Cet is the only one that has a ratio that includes some phase-dependent effects and is slightly smaller than unity.

The ratio between the $K 2.26$ and $H 1.65$ filters has a more pronounced pulsation phase signature (see Fig. 11, middle panel), which reflects more complex and disjoint temperature and opacity changes between these two layers. The ratio reaches its minimum (i.e., $H 1.65 \mathrm{UD}>K 2.26 \mathrm{UD}$ ) before minimum light and its maximum at maximum light. In order to compare our data with the observations of Millan-Gabet et al. (2005), we also calculated the mean diameter ratio to be

$$
\overline{R_{K 2.26 / H 1.65}}=1.11 \pm 0.11,
$$

a mean ratio marginally larger than the $H 1.65 / J 1.24$ mean ratio. Again, our data agree, to within errors, with the value of $\overline{R_{K^{\prime} / H}}=$ $1.12 \pm 0.09$ reported by Millan-Gabet et al. (2005).

As mentioned earlier, the $L 3.08$ angular diameter behaves differently. It reaches minimum values at minimum light and the best fit to the diameter pulsation is shifted by 0.5 cycles compared with the $H 1.65$ pulsation, albeit at a much smaller relative pulsation amplitude. As the bottom panel of Figure 11 shows, this phase shift and the UD diameter ratio between the two layers seem to be similar for all Mira variables in our sample (including
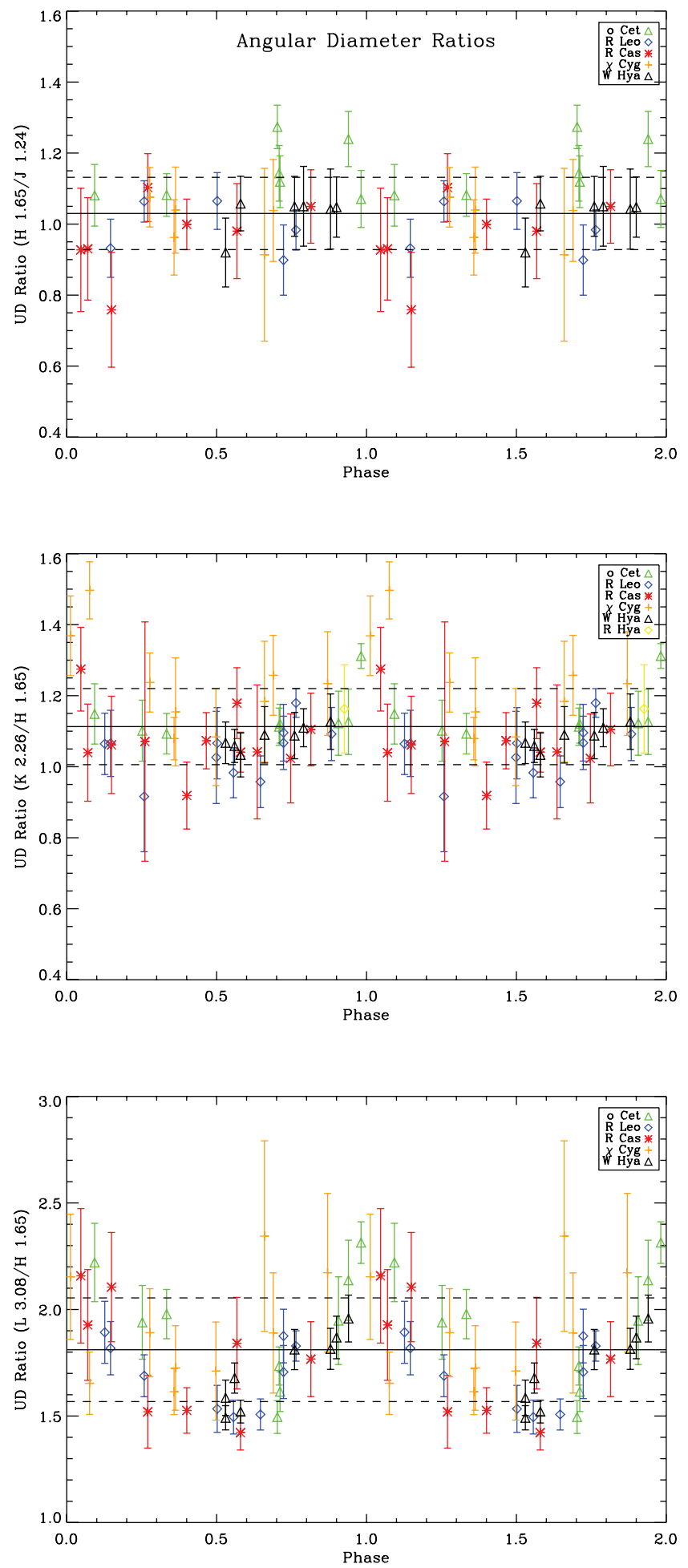

FIG. 11.-Angular diameter ratios for all Mira variables in this study. The solid lines indicate the mean ratio, and the dashed lines indicate the standard deviation of the sample. The data are repeated to show two cycles for better recognition of the pulsation pattern.

$\chi$ Cyg). For consistency sake, we calculated the mean diameter ratio to be

$$
\overline{R_{L 3.08 / H 1.65}}=1.81 \pm 0.24 \text {. }
$$

This unusual UD diameter variation has never been observed before and raises questions about the mechanism of the observed 
pulsation in the $L 3.08$ layer. The $L 3.08$ light curve follows a similar trend to the $J H K$ light curves, possibly indicating a temperature devolution similar to the lower layers, and as the inner layers of the star are shrinking and heating up, the outer layers are either expanding or becoming increasingly opaque. Whether opacity effects or dynamic motion of these outer layers (or both) are responsible for this surprising behavior is the subject for model interpretations and will be the subject of a subsequent study.

\subsection{Individual Stars}

Of the six Mira variables observed, four have observational phase coverage suitable for further comparisons with pulsation models ( $o$ Cet, R Leo, R Cas, and $\chi$ Cyg). The Mira variables studied differ substantially in behavior regarding pulsation amplitudes, diameter-wavelength relationships, and diameter-phase relationships.

In this section we discuss the results of this study for each individual star, emphasizing the main differences and similarities found in this subset.

$o$ Cet.-The prototype of Mira stars is one of the most observed variable stars, due to its brightness, amplitude $(V \approx 3-10$; A. A. Henden et al. 2006, private communication), and closeness $(107 \pm 6$ pc; Knapp et al. 2003). Its size in different bandwidths (e.g., Haniff et al. 1995; Mennesson et al. 2002; Woodruff et al. 2004), optical spectra (Joy 1954), light curves in different colors (e.g., Whitelock et al. 2000; Nadzhip et al. 2001; AAVSO), asymmetries (e.g., Karovska et al. 1991; Tuthill et al. 1999), and companion star (e.g., Karovska et al. 1997; Wood \& Karovska 2006; Ireland et al. 2007) have been subject to intense research.

Figure 4 shows the UD diameter variation in the $J 1.24, H 1.65$, and $K 2.26$ bandpasses as nearly synchronous (within the 0.1 phase shift shown in Fig. 10), sinusoidal pulsations, with an apparent phase shift to the diameter pulsation in the $L 3.08$ bandpass. The UD diameter versus phase curves agree well with the fitted sine functions, with reduced $\chi^{2}$ for $J 1.24, H 1.65, K 2.26$, and $L 3.08$ having the values $1.45,1.17,1.02$, and 0.80 , respectively. The shift of $\approx 0.5$ cycles between the sine curves fitted to the diameter versus phase in the $H 1.65$ and the $L 3.08$ bandpasses has never been observed or predicted, and it can also be observed in R Leo, R Cas, and W Hya. A similar shift, although not as pronounced, can be seen in $\chi$ Cyg.

The $K 2.26$ UD angular diameter values lie in the range of $31.0 \pm 1.9$ mas near $V$ maximum and $37.0 \pm 1.2$ mas at phase 0.7 . These values agree with the interferometric UD diameters within the $K$ band of Ridgway et al. (1992) (phase 0.8) and Woodruff et al. (2004) (phases 0.1-0.4; see Table 10). Our UD angular diameters are generally larger than those measured by Mennesson et al. (2002) (phases 0.9-0.0). This is most likely due to the larger spatial frequencies (i.e., longer baselines) at which their measurements were made, combined with the known departure of $o$ Cet's brightness distribution from UD (cf. Woodruff et al. 2004). Our $K 2.26$ UD diameters are also larger than the molecular layer diameters obtained by Perrin et al. (2004) $(24.95 \pm$ 0.10 to $26.84 \pm 0.06$ mas) at similar phases by fitting ad hoc scenarios (a photosphere surrounded by an emissive and absorbing layer) to $K$ and $L^{\prime}$ interferometric data. This is to be expected, as their visibilities also show obvious departures from simple models, and fitting the same data to a brightness distribution consisting of a central object with a bright molecular shell would yield smaller diameters than a UD fit. Note that these very simple scenarios are not always unique and a new ad hoc parameter set has to be determined for each observation, making a comparison awkward. Star $o$ Cet shows little cycle-to-cycle variation through- out our data, although this could be attributed to the observing of a stable era of its pulsation and might have been different if observed, e.g., $10 \mathrm{yr}$ earlier.

The $L 3.08 \gg K 2.26>H 1.65>J 1.24$ layering of monochrome diameters is strictly monotonous, unlike, e.g., R Leo, where the sequence of UD diameters seems to invert during the pulsation cycle. The fact that $o$ Cet appears largest in the $J H K$ filters approximately at visual phase 0.6 , roughly coinciding with the minimum of the NIR and visual light curves ( $\operatorname{see} \S 3.1$ ), is in accordance with various model interpretations (e.g., Jacob \& Scholz 2002; Ireland et al. 2004a, 2004b; Ireland \& Scholz 2006). The unusual behavior of the $L 3.08$ layer, as described in $\S 4.2$, will be further discussed in $\S 5$ and in a follow-up paper.

$R$ Leo.-Although $\mathrm{R}$ Leo has a similar period (310 days), $V$ magnitude range (4.4-11.3; Kholopov et al. 1998), and spectral type as $o$ Cet, we found significant differences in this study. The pulsation amplitudes of layers in different bandpasses and their phases with respect to one another seem to show a more complex trend than $o$ Cet's atmosphere. The pulsation is less pronounced than $o$ Cet's in the NIR, with UD diameters varying between $29.6 \pm 1.4$ and $33.1 \pm 2.0$ mas $(\approx 12 \%)$ in the $J 1.24$ bandpass, which shows the largest relative pulsation amplitude. There is an overall agreement with cited UD radii except for the long-baseline UD angular diameters of Fedele et al. (2005). This can be due to the same effect as described above for $o$ Cet concerning the long-baseline measurements of Mennesson et al. (2002). With our H 1.65 measurements we are able to disambiguate the two possible solutions (due to ambiguous model fitting) for the $H$-band diameter found by Millan-Gabet et al. (2005). Due to the seeming lack of cycle-to-cycle dependence in this bandpass, we can reject their smaller angular diameter of $23.8 \pm 0.3$ mas at phase 0.4 and substantiate their larger diameter of $32.4 \pm 0.4$ mas.

The variation between $47.8 \pm 1.8$ and $56.5 \pm 2.0 \operatorname{mas}(\approx 18 \%)$ in the $L 3.08$ bandpass shows a steady increase in diameter between 1997 December 16 and 2001 June 11 and no significant diameter change up to three pulsation cycles later. This gradual increase in angular diameter over four cycles may be understandable in terms of the noncyclic time evolution of positions of outer mass zones of pulsation models over several successive cycles (see Fig. 1 of Ireland et al. 2004a, 2004b), which affect the position and physics of water shells. This interpretation is supported by the steady brightening of the visual photometry maxima during the same four cycles in which the UD diameter increases, indicating variability in timescales longer than the pulsation phase. The pulsation signature of the $L 3.08$ bandpass shows nonetheless the general $\approx 0.5$ phase lag compared to the $H 1.65$ band, albeit with a larger uncertainty in the least-squares sinusoidal fit (reduced $\chi^{2}=1.38$ ).

$R$ Cas.-Of the two Mira variables with the longest periods in our sample, R Cas (430 days) also exhibits the latest spectral type. In fact, at minimum visual pulsation phase it defines the spectral type M10 (see Lockwood \& Wing 1971). It seems to follow the model-predicted phase-diameter trend of larger diameters around minimum light in the $H 1.65$ filter, albeit with some cycle-to-cycle uncertainty. The scatter is considerably greater in the $J 1.24$ and $K 2.26$ filters, but the same trend is still present. Note that the relative phase shift of diameter maxima from shorter to longer wavelengths in $J H K$ is very distinctive, even when the uncertainties introduced by cycle-to-cycle variations in the diameter pulsations are taken into account. The position of the $L 3.08$ layer also follows the pattern of the other M-type Mira variables, in that it seems to pulsate with a 0.5 phase 
TABLE 10

Published Interferometric Ud Angular Diameters in the $K$ Band for the Stars in This Study

\begin{tabular}{|c|c|c|c|c|c|}
\hline Star & Reference & $\begin{array}{l}\text { Angular Diameter } \\
\text { (mas) }\end{array}$ & $\begin{array}{c}\text { Center Wavelength } \\
\qquad(\mu \mathrm{m})\end{array}$ & $\begin{array}{l}\text { Bandwidth } \\
\quad(\mu \mathrm{m})\end{array}$ & Visual Phase \\
\hline \multirow[t]{11}{*}{$o$ Cet........................... } & 1 & $36.1 \pm 1.4$ & 2.2 & 0.4 & 0.8 \\
\hline & 2 & $28.79 \pm 0.10$ & 2.2 & 0.44 & 0.9 \\
\hline & 2 & $25.73 \pm 0.09$ & 2.03 & $\approx 0.1$ & 0.0 \\
\hline & 2 & $25.13 \pm 0.08$ & 2.15 & $\approx 0.1$ & 0.0 \\
\hline & 2 & $25.19 \pm 0.12$ & 2.22 & $\approx 0.1$ & 0.0 \\
\hline & 2 & $29.22 \pm 0.12$ & 2.39 & $\approx 0.1$ & 0.0 \\
\hline & 2 & $24.40 \pm 0.11$ & 2.16 & 0.32 & 0.0 \\
\hline & 3 & $29.24 \pm 0.30$ & 2.2 & 0.4 & 0.1 \\
\hline & 3 & $29.53 \pm 0.30$ & 2.2 & 0.4 & 0.2 \\
\hline & 3 & $30.49 \pm 0.30$ & 2.2 & 0.4 & 0.3 \\
\hline & 3 & $33.27 \pm 0.33$ & 2.2 & 0.4 & 0.4 \\
\hline \multirow[t]{8}{*}{ R Leo } & $4^{\mathrm{a}}$ & $33.0 \pm 1.3$ & 2.16 & 0.03 & 0.2 \\
\hline & 5 & $28.18 \pm 0.05$ & 2.16 & 0.32 & 0.2 \\
\hline & 5 & $30.68 \pm 0.05$ & 2.16 & 0.32 & 0.3 \\
\hline & $6^{\mathrm{a}}$ & $34 \pm 2$ & 2.36 & 0.46 & 0.4 \\
\hline & 7 & $30.3 \pm 0.3$ & $2.26,2.16$ & $0.05,0.32$ & $0.6-0.7$ \\
\hline & 8 & $29.91 \pm 0.27$ & 2.16 & 0.32 & 0.4 \\
\hline & 9 & $28.1 \pm 0.05$ & 2.2 & 0.4 & 0.1 \\
\hline & 9 & $26.2 \pm 0.01$ & 2.2 & 0.4 & 0.0 \\
\hline 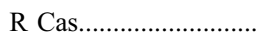 & 10 & $22.03_{-4.14}^{+2.13}$ & 2.2 & 0.4 & 0.8 \\
\hline \multirow[t]{2}{*}{ 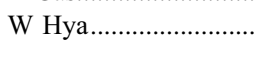 } & 7 & $42.5 \pm 0.7$ & $2.26,2.16$ & $0.05,0.32$ & 0.5 \\
\hline & 8 & $39.9 \pm 0.2$ & 2.16 & 0.32 & 0.6 \\
\hline 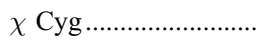 & 2 & $23.24 \pm 0.08$ & 2.16 & 0.32 & 0.38 \\
\hline \multirow[t]{2}{*}{ R Нya .............................. } & 8 & $23.9 \pm 0.5$ & 2.16 & 0.32 & 0.8 \\
\hline & 8 & $25.8 \pm 0.2$ & 2.16 & 0.32 & 0.8 \\
\hline
\end{tabular}

${ }^{a}$ Obtained by lunar occultation.

References.-(1) Ridgway et al. 1992; (2) Mennesson et al. 2002; (3) Woodruff et al. 2004; (4) di Giacomo et al. 1991; (5) Perrin et al. 1999; (6) Tej et al. 1999; (7) Monnier et al. 2004; (8) Millan-Gabet et al. 2005; (9) Fedele et al. 2005; (10) van Belle et al. 2002.

shift to the $H 1.65$ layer. Van Belle et al. (2002) measured a $K$ UD angular diameter of $22.03_{-4.14}^{+2.13}$ mas at phase 0.81 , which does not agree with our diameter of $28.9 \pm 1.9$ mas at the same pulsation phase but agrees with the diameter measured at roughly the same phase in a different cycle $(26.1 \pm 2.1$ mas at phase $0.74)$, another indicator of noticeable cycle-to-cycle variation.

W Hya.-At a distance of $78 \pm 3$ pc (Knapp et al. 2003), W Hya is the closet and best-resolved Mira in our sample. We observe a relatively time-independent $K 2.26$ angular diameter of $\approx 40 \pm 5$ mas in the phase range $\Phi=0.53-0.88$. Because of W Hya's low declination and proximity to the ecliptic, and because the period is close to $1 \mathrm{yr}$, the phase coverage only spans half the pulsation cycle (see Table 7). The previously measured UD angular diameters in the $K$ band by Monnier et al. (2004) and Millan-Gabet et al. (2005) (see Table 10) are consistent with our measurements. The $H$-band diameter of Millan-Gabet et al. (2005) (31.3 $\pm 0.3 \mathrm{mas})$ is slightly smaller than our derived $H 1.65$ UD angular diameters, which is most likely due to the same effects of long-baseline interferometry as described for $o$ Cet and R Leo. Although all Mira intensity distributions show deviations from a UD profile, we can clearly detect those only in W Hya. This could be due to the presence of dust emission and/or the partially resolved brightness distribution of extended molecular layers in the upper atmosphere.

The largest $L 3.08$ angular diameter is found around maximum visual light, where the $J 1.24$ and $H 1.65$ diameters are smallest.

$\chi C y g$.-The Mira in our sample with the second longest pulsation period, $\chi$ Cyg (408 days) is the only S-type Mira, albeit with M-type characteristics (cf. Keenan \& Boeshaar 1980). It deviates noticeably from the M-type Mira variables in many ways. The visual light curve of $\chi$ Cyg is well known for its particularly large magnitude range, and its NIR colors are also all unusually red, as observed by Whitelock et al. (2000). The $L$ 3.08 mean magnitude, in particular, is comparable to or brighter than that of $K 2.26$, whereas for all other Mira variables in our study the $L 3.08$ magnitudes lie in the range between the $H 1.65$ and the $K 2.26$ light curves.

The relative diameter pulsation amplitude is larger in all filters than that of the other Mira variables in this paper, and the derived UD angular diameters show small cycle-to-cycle variation. There are no model predictions for S-type Mira variables to date, and we can only speculate whether or not this behavior is related to the fundamental stellar parameters or to the fact that differing C-to-O ratio led to significant changes in $\mathrm{H}_{2} \mathrm{O}$ formation characteristics (cf. Ohnaka 2004) and more stable opacity structures.

While the $L 3.08$ UD diameter pulsation follows the same trend as $o$ Cet, R Leo, R Cas, and W Hya, in that it reaches maximum amplitude around maximum visual light, the $J H K$ diameters show a somewhat different behavior. Note that the shapes of the visibility curves deviate increasingly from a UD profile with increasing wavelength. The $K 2.26$ and $H 1.65$ UD angular diameters reach their maximum values just after maximum light, at phase $0.1-0.2$, coinciding with the maximum of the correspondent light curves. This may indicate a heavy contamination of these filters by molecular layers in the star's atmosphere. On the other hand, the $J 1.24$ band data infer a smaller 
UD diameter with its maximum around minimum light, as observed in the other Mira variables in our sample, suggesting a deeper view into $\chi$ Cyg's atmosphere.

Young et al. (2000) only detected a slight variation in the $J$-band angular diameters, albeit over a very small phase coverage. If we fit a sine curve to our derived $J 1.24$ angular diameters, then we find that Young et al. (2000) measurements lie roughly symmetrically around the diameter pulsation minimum, a factor that could explain the lack of diameter variation in their studies. We converted the $J$-band Gaussian FWHM from Young et al. (2000) to UD angular diameters using the UD-to-Gaussian ratio $R \approx 1.5$ calculated by Burns et al. (1998) and find that the diameters approximately match our simple sine curve predictions. Mennesson et al. (2002) report a $K^{\prime}$ (centered at $2.16 \mu \mathrm{m}$, $0.32 \mu \mathrm{m}$ wide) UD angular diameter of $23.24 \pm 0.08$ mas for $\chi$ Cyg on 2000 May, at variable phase 0.38. In June of the same year (phase 0.48 ) we obtained a $K 2.26$ diameter of $25.0 \pm$ 2.2 mas, in reasonable agreement. In the next two subsequent years (both at phase 0.36 ) we observed UD diameters of approximately 30 mas, an increase in diameter of $20 \%$ over one cycle, which might be linked to transient opacity structures.

\section{SUMMARY}

We have measured the diameters and NIR light curves of six Mira variables stars at up to 19 separate phases in four filters, the first study of this magnitude. We present the first narrowband $3.08 \mu \mathrm{m}$ light curves of Mira variables. The NIR light curves can be approximated by a sine function and confirm the phase shift of $\approx 0.15-0.22$ by which the NIR maxima lag behind the visual maxima previously reported by Nadzhip et al. (2001) and Smith et al. (2002). In addition, we find an NIR photometric flux relation: $J 1.24<H 1.65<K 2.26$, in agreement with the $J H K$ observations of Whitelock et al. (2000) and Smith et al. (2002). The $L 3.08$ fluxes in our sample are slightly less than or equal to the $K 2.26$ fluxes, except for $\chi$ Cyg, where the $L 3.08$ magnitudes are comparable to the ones in the $K 2.26$ bandpass.

We found no correlation between NIR photometry and UD diameter cycle-to-cycle variations, yet we found some correlation between the $L 3.08$ UD diameters and the visual light curves (see the discussion of R Leo in $\S 4.3$ ). All observed stars show variations of their UD angular diameters as a function of pulsation phase. We find the UD diameter relation $J 1.24<H 1.65<$ $K 2.26$ to be an average value only, with deviations throughout the pulsation cycle, revealing the complexity of phase-dependent opacity contamination from molecules in different layers. Of the Mira variables in our sample, only $o$ Cet shows this strict layering throughout its cycle, in agreement with theoretical models designed to represent $o$ Cet (see Jacob \& Scholz 2002). The pulsation amplitude also does not follow the model-predicted dependency on molecular opacity (Ireland et al. 2004a, 2004b). The layers exhibiting the largest relative variation in UD diameter $(6 \%-18 \%)$ were those seen through the $H 1.65$ and $J 1.24$ filters, which should display less molecular contamination and thus less diameter variation. The $K 2.26$ layer has UD diameter pulsation amplitudes between $4 \%$ and $7 \%$. The molecular layer probed by the $L 3.08$ bandpass is significantly farther from the photosphere than the $J H K$ layers. It varies in UD angular diameter by as little as $4 \%$ and as much as $8 \%$, with a 0.5 phase offset to the $H 1.65$ pulsation, and is between 1.5 and 2.5 times larger than the $H 1.65$ UD angular diameter, depending on the pulsation phase. This behavior has not been observed before, and detailed models are needed to understand it.

Another quantity predicted by models are the diameters in different wavelengths at different phases. When comparing the two stars whose parameters are thought to resemble the model input parameters the most, R Leo and $o$ Cet (both with revised Hipparcos parallaxes; see Knapp et al. 2003), with model predictions (Ireland et al. 2004a, 2004b), we find that our UD diameters are significantly too large in the $K 2.26$ bandpass. This could be explained by too high model effective temperatures, but a more careful, model-based interpretation is needed to understand this effect. Given the small baselines $(<10 \mathrm{~m})$ used in this experiment, it is remarkable how consistent the data are for the M-type Mira variables. It is even more surprising how different the S-type Mira $\chi$ Cyg appears to be when its multiwavelength pulsation signature is analyzed. The $H 1.65$ and $K 2.26$ UD angular diameters are smallest around minimum light, contrary to all models and previous observations of M-type Mira variables. Further work with these data, including model comparisons with individual stars, imaging, and asymmetry studies, will be presented in subsequent publications.

The visibility data presented in this publication are available on request.

This work has been supported by grants from the National Science Foundation, the Australian Research Council, and the Deutsche Forschungsgemeinschaft (H. C. W., M. S.). The data presented herein were obtained at the W. M. Keck Observatory, which is operated as a scientific partnership among the California Institute of Technology, the University of California, and the National Aeronautics and Space Administration. The Observatory was made possible by the generous financial support of the W. M. Keck Foundation. We acknowledge with thanks the variable star observations from the AAVSO International Database contributed by observers worldwide and used in this research. We also thank Albert Jones and Peter Williams for the W Hya light-curve data.
Aringer, B., Kerschbaum, F., \& Jörgensen, U. G. 2002, A\&A, 395, 915 Aufdenberg, J. P., et al. 2006, ApJ, 645, 664

Bessell, M. S., Scholz, M., \& Wood, P. R. 1996, A\&A, 307, 481

Burns, D., et al. 1998, MNRAS, 297, 462

Danchi, W. C., Bester, M., Degiacomi, C. G., Greenhill, L. J., \& Townes, C. H. 1994, AJ, 107, 1469

di Giacomo, A., Lisi, F., Calamai, G., \& Richichi, A. 1991, A\&A, 249, 397

Dumm, T., \& Schild, H. 1998, NewA, 3, 137

Dyck, H. M., Benson, J. A., van Belle, G. T., \& Ridgway, S. T. 1996, AJ, 111, 1705

Dyck, H. M., van Belle, G. T., \& Thompson, R. R. 1998, AJ, 116, 981

Fedele, D., Wittkowski, M., Paresce, F., Scholz, M., Wood, P. R., \& Ciroi, S. 2005, A\&A, 431, 1019

Golay, M. J. E. 1971, J. Opt. Soc. Am., 61, 272

Haniff, C. A., Scholz, M., \& Tuthill, P. G. 1995, MNRAS, 276, 640

\section{REFERENCES}

Hofmann, K.-H., Scholz, M., \& Wood, P. R. 1998, A\&A, 339, 846

Hofmann, K.-H., et al. 2000, Proc. SPIE, 4006, 688 2002, NewA, 7, 9

Ireland, M. J. 2006, Proc. SPIE, 6268, 10

Ireland, M. J., \& Scholz, M. 2006, MNRAS, 367, 1585

Ireland, M. J., Scholz, M., Tuthill, P. G., \& Wood, P. R. 2004a, MNRAS, 355, 444

Ireland, M. J., Scholz, M., \& Wood, P. R. 2004b, MNRAS, 352, 318

Ireland, M. J., Tuthill, P. G., Bedding, T. R., Robertson, J. G., \& Jacob, A. P. 2004c, MNRAS, 350, 365

Ireland, M. J., et al. 2007, ApJ, 662, 651

Jacob, A. P., \& Scholz, M. 2002, MNRAS, 336, 1377

Joy, A. H. 1954, ApJS, 1, 39

Jura, M., \& Kleinmann, S. G. 1990, ApJS, 73, 769 
Karovska, M., Hack, W., Raymond, J., \& Guinan, E. 1997, ApJ, 482, L175 Karovska, M., Nisenson, P., Papaliolios, C., \& Boyle, R. P. 1991, ApJ, 374, L51

Keenan, P. C., \& Boeshaar, P. C. 1980, ApJS, 43, 379

Kholopov, P. N., et al. 1998, Combined General Catalogue of Variable Stars

Knapp, G. R., Pourbaix, D., Platais, I., \& Jorissen, A. 2003, A\&A, 403, 993

Lançon, A., \& Wood, P. R. 2000, A\&AS, 146, 217

Lockwood, G. W., \& Wing, R. F. 1971, ApJ, 169, 63

Mennesson, B., et al. 2002, ApJ, 579, 446

Millan-Gabet, R., Pedretti, E., Monnier, J. D., Schloerb, F. P., Traub, W. A., Carleton, N. P., Lacasse, M. G., \& Segransan, D. 2005, ApJ, 620, 961

Monnier, J. D. 1999, Ph.D. thesis, Univ. California, Berkeley

Monnier, J. D., Tuthill, P. G., \& Danchi, W. C. 2002, ApJ, 567, L137

Monnier, J. D., et al. 2004, ApJ, 605, 436

Mozurkewich, D., et al. 1991, AJ, 101, 2207 . 2003, AJ, 126, 2502

Nadzhip, A. E., Tatarnikov, A. M., Shenavrin, V. I., Weigelt, G., \& Yudin, B. F. 2001, Astron. Lett., 27, 324

Ohnaka, K. 2004, A\&A, 424, 1011

Percy, J. R., \& Au, W. W.-Y. 1999, PASP, 111, 98

Perrin, G., Coudé du Foresto, V., Ridgway, S. T., Mariotti, J.-M., Traub, W. A., Carleton, N. P., \& Lacasse, M. G. 1998, A\&A, 331, 619

Perrin, G., Coudé du Foresto, V., Ridgway, S. T., Mennesson, B., Ruilier, C., Mariotti, J.-M., Traub, W. A., \& Lacasse, M. G. 1999, A\&A, 345, 221

Perrin, G., et al. 2004, A\&A, 426, 279

Ragland, S., et al. 2006, ApJ, 652, 650

Richichi, A., \& Percheron, I. 2002, A\&A, 386, 492

Richichi, A., Percheron, I., \& Khristoforova, M. 2005, A\&A, 431, 773

Ridgway, S. T., Benson, J. A., Dyck, H. M., Townsley, L. K., \& Hermann, R. A. 1992, AJ, 104, 2224
Ridgway, S. T., Wells, D. C., Joyce, R. R., \& Allen, R. G. 1979, AJ, 84, 247 Scholz, M. 2003, Proc. SPIE, 4838, 163

Scholz, M., \& Wood, P. R. 2000, A\&A, 362, 1065

Sloan, G. C., \& Price, S. D. 1998, ApJS, 119, 141

Smith, B. J., Leisawitz, D., Castelaz, M. W., \& Luttermoser, D. 2002, AJ, 123, 948

Tej, A., Chandrasekhar, T., Ashok, N. M., Ragland, S., Richichi, A., \& Stecklum, B. 1999, AJ, 117, 1857

Tej, A., Lançon, A., Scholz, M., \& Wood, P. R. 2003, A\&A, 412, 481

Thompson, R. R., Creech-Eakman, M. J., \& van Belle, G. T. 2002, ApJ, 577, 447

Tuthill, P. G., Danchi, W. C., Hale, D. S., Monnier, J. D., \& Townes, C. H. 2000a, ApJ, 534, 907

Tuthill, P. G., Haniff, C. A., \& Baldwin, J. E. 1995, MNRAS, 277, 1541 1999, MNRAS, 306, 353

Tuthill, P. G., Monnier, J. D., Danchi, W. C., Hale, D. D. S., \& Townes, C. H. 2002, ApJ, 577, 826

Tuthill, P. G., Monnier, J. D., Danchi, W. C., Wishnow, E. H., \& Haniff, C. A. 2000b, PASP, 112, 555

van Belle, G. T., Dyck, H. M., Benson, J. A., \& Lacasse, M. G. 1996, AJ, 112, 2147

van Belle, G. T., Thompson, R. R., \& Creech-Eakman, M. J. 2002, AJ, 124, 1706

Whitelock, P., Marang, F., \& Feast, M. 2000, MNRAS, 319, 728

Wood, B. E., \& Karovska, M. 2006, ApJ, 649, 410

Wood, P. R., et al. 1999, in IAU Symp. 191, Asymptotic Giant Branch Stars, ed. T. Le Bertre, A. Lebre, \& C. Waelkens (San Francisco: ASP), 151

Woodruff, H. C., et al. 2004, A\&A, 421, 703

Young, J. S., et al. 2000, MNRAS, 318, 381 